Silva et al.

\title{
In situ and transcriptomic identification of synapse-associated microglia in the developing zebrafish brain
}

Authors: Nicholas J. Silva ${ }^{1}$, Leah C. Dorman ${ }^{1,2}$, Ilia D. Vainchtein ${ }^{1}$, Nadine C. Horneck ${ }^{1}$, and Anna V. Molofsky ${ }^{1,5 *}$

Affiliations:

${ }^{1}$ Department of Psychiatry and Behavioral Sciences/Weill Institute for Neurosciences, ${ }^{2}$ Neuroscience Graduate Program, ${ }^{2}$ Department of Laboratory Medicine, ${ }^{5}$ Kavli Institute for Fundamental Neuroscience, University of California, San Francisco, San Francisco, CA.

\section{Twitter handles:}

@NeuroNiko (Nicholas Silva)

@sculptorofdance (Leah Dorman)

@IliaVainchtein (Ilia Vainchtein)

@AnnaMolofskyLab (Anna Molofsky)

${ }^{*}$ Correspondence to:

Anna V Molofsky MD PhD, University of California San Francisco, $15504^{\text {th }}$ Street, San Francisco, CA 94158, USA. Tel: 1 (415) 502-3609. anna.molofsky@ucsf.edu, @AnnaMolofskyLab

\section{Summary}

Microglia are brain resident macrophages that play vital roles in central nervous system (CNS) development, homeostasis, and pathology. Microglia both remodel synapses and engulf apoptotic cell corpses during development, but whether unique molecular programs regulate these distinct phagocytic functions is unknown. Here we identify a molecularly distinct synapse-associated microglial subset in the zebrafish (Danio rerio). We found that ramified microglia populated synapse-rich regions of the midbrain and hindbrain between 7 and 28 days post fertilization. In contrast, microglia in the optic tectum were ameboid and clustered around neurogenic zones. Using single-cell mRNA sequencing combined with metadata from regional bulk sequencing, we identified synapse-associated microglia (SAMs) that were highly enriched in the hindbrain, expressed known synapse modulating genes as well as novel candidates, and engulfed synaptic proteins. In contrast, neurogenic-associated microglia (NAMs) were enriched in optic tectum, had active cathepsin activity, and preferentially engulfed neuronal corpses. These data yielded a functionally annotated atlas of zebrafish microglia (https://www.annamolofskylab.org/microgliasequencing). Furthermore, they reveal that molecularly distinct phagocytic programs mediate synaptic remodeling and cell engulfment, and establish zebrafish hindbrain as a model circuit for investigating microglial-synapse interactions. 
Silva et al.

\section{Introduction}

Microglia, the dominant immune cells in the central nervous system (CNS), perform critical and diverse functions during brain development and disease, including engulfing synapses, promoting synapse formation, and clearing apoptotic neurons, ${ }^{1,2}$. However, it is not clear whether these diverse functions are mediated by molecularly distinct subsets of microglia. Single-cell sequencing of mouse microglia reveals transcriptional heterogeneity predominantly during development ${ }^{3,4}$, whereas functional studies reveal region-specific microglial populations that persist into adulthood ${ }^{5-7}$. These data suggest that transcriptional profiling in rodents has not yet been able to resolve known functional heterogeneity in microglia. Furthermore, linking these transcriptionally identified subpopulations to functional subsets in situ remains challenging. Thus, despite abundant evidence that microglia both modulate synapses and engulf cell corpses during development, the molecular regulation of these functions is not well understood.

The zebrafish (Danio rerio) is an increasingly utilized vertebrate model for developmental neuroscience ${ }^{8}$ that has not been used to study microglial-synapse interactions. Zebrafish contain ontogenetically similar microglia ${ }^{9-11}$, as well as other glial cell types ${ }^{12-14}$. Fish have a highly homologous immune system to mammals ${ }^{15-17}$, including meningeal lymphatics ${ }^{18}$ and a blood brain barrier that matures between 8-10 dpf ${ }^{19}$. Fish nervous system development also resembles mammals, with the exception that robust neurogenesis persists throughout life, leading to ongoing turnover of neuronal corpses in neurogenic regions, particularly in the midline optic tectum (OT). Much of our functional understanding of fish microglia comes from elegant work in the OT, which has identified molecular mechanisms that drive phagocytosis of neuronal corpses ${ }^{20-22}$. However, in the OT are functionally and ontogenetically distinct from microglia in other CNS regions ${ }^{23-26}$. Furthermore, a distinct subset of microglia in the spinal cord white matter engulfs myelin sheaths ${ }^{27}$, and is linked to leukodystrophy ${ }^{28}$.

These regionally and functionally distinct microglial subsets raise the question of whether synapse-associated microglia also exist in the fish, and if so, whether they play a functional role in CNS development. Bulk transcriptomic sequencing in the fish has begun to uncover key information regarding microglial ontogeny, revealing that microglia populate the CNS in two waves 29,30. An embryonic wave derived from the rostral blood island, similar to the yolk sac of mammals, persists for the first two months and is later replaced by an adult population derived from ventral wall of dorsal aorta (VDA) ${ }^{29}$. Recent bulk sequencing studies describe microglia transcriptional heterogeneity in the adult zebrafish brain, linked to ontogeny, that correlates with differing amounts of bacterial phagocytosis in vitro ${ }^{31}$. However, most canonical microglial genes identified in mice and humans ${ }^{32}$ are conserved in both developmental waves of zebrafish microglia ${ }^{33-35}$. Like in mammals, it is not clear to what extent ontogeny impacts function, thus a focus on linking the functional transcriptome to in vivo function is essential.

Here we define a distinct subpopulation of synapse-associated microglia (SAMs) in the juvenile fish using in situ characterization as well as single cell and bulk RNA sequencing. SAMs were ramified and expanded in the midbrain and hindbrain after 7 days post fertilization (dpf). These microglia engulf neuronal synaptic proteins as in mammals, and were defined by expression of complement genes (C1qa, C1qc) and other novel candidate pathways. In contrast, microglia in the optic tectum clustered near neurogenic regions, were rich in lysosomal gene expression (Ctsla, Ctsba), and their phagocytic capacity correlated with functional cathepsin activity. These data define a molecular profile associated with phagocytosis of synaptic proteins and define a novel model system in which to study microglial-synapse interactions. 
Silva et al.

\section{Results:}

\section{Synapse-associated microglia expand developmentally in the zebrafish hindbrain and are distinct from neurogenic-associated microglia}

To characterize microglia in both synaptic and neurogenic regions of the fish brain, we performed immunohistochemistry using the myeloid reporter line $T g(m p e g: E G F P){ }^{36}$. We used the presynaptic vesicle marker SV2 to demarcate synapse rich regions, and BrdU to identify neurogenic zones. We found that microglia colocalize with synapse rich regions of the midbrain and hindbrain as early as 7 days post fertilization (dpf) and increased in number over the late larval to juvenile period (14-28 dpf; Fig. 1A, B). By 28 dpf most microglia in the midbrain and hindbrain were colocalized with the presynaptic marker, SV2 (65\% and 80\%, respectively; Fig. 1C,D). In contrast, $>60 \%$ of microglia in the optic tectum (OT) clustered near the BrdU+ neurogenic zone (Fig. 1E,F), consistent with their known roles in eliminating apoptotic cell corpses ${ }^{24,26}$. Immunostaining with the commonly used microglia antibody $4 \mathrm{C} 4$ were consistent with these findings and indicated that the majority (70-90\%) of mpeg:EGFP+ cells in the brain are microglia, with a modestly lower proportion in the midbrain and hindbrain relative to OT (Fig. S1AD). We next characterized microglial morphology in hindbrain and OT. We found that microglia (mpeg:EGFP+4C4+) in the hindbrain were ramified and more closely resembled microglia in the postnatal rodent brain, whereas OT microglia were significantly more ameboid, as quantified by increased sphericity (Fig. 1G,H). In summary, we identified a ramified synapse-associated microglia (SAM) enriched in the hindbrain and midbrain that was distinct from amoeboid neurogenic-associated microglia (NAM) in the optic tectum.

\section{Molecularity distinct subsets of microglia identified at single cell resolution during brain development}

To determine whether microglia in the zebrafish brain are molecularly heterogeneous, we performed single-cell RNA sequencing. We flow sorted hematopoietic cells from juveniles at 28 dpf using a CD45:DsRed reporter ${ }^{37}$ crossed with myeloid-specific mpeg:eGFP to ensure that all potential subsets of immune cells were captured (Fig. 2A, S2A-B). Our juvenile time-point at 28 dpf encompasses both developmental waves of embryonic and adult microglia in the zebrafish brain ${ }^{29,30}$. Flow analysis and our initial single-cell clustering indicated that $90 \%$ of CD45+ cells were mpeg-EGFP positive, and that the CD45+ population captured all mpeg:eGFP positive cells. Unbiased clustering of CD45+ cells revealed 15 distinct clusters of hematopoietic origin, including seven non-myeloid clusters (mpeg ${ }^{\text {neg }}$;Fig. 2B, S2B-C; Table S1). These included clusters that expressed markers for T-cells (cd4-1, Ick, and ccr9a), natural killer cells (eomesa), and innate lymphocyte-like cells (il13, gata3) ${ }^{38-41}$; Fig. S2C). Thus, multiple immune cell subsets, predominately myeloid in origin, are present in the zebrafish brain, although it is possible that some of these may be circulating rather than tissue-resident.

To focus on myeloid cells, which are the dominant immune cell subset, we reclustered 3539 mpeg+ cells, which after quality control and filtering, yielded six distinct myeloid clusters (Fig. 2C; Fig. S2D-F; Table S2). We identified cluster JM3 as the macrophage subset based on the absence of microglial-specific markers (p2ry12, csf1rb, hexb, and slc7a7) and the presence of ccl19a.1 (a.k.a. macrophage inflammatory protein; Fig. 2D) and higher levels of mpeg:EGFP; Fig. S2G). Cluster JM2 marked a proliferating subset (pcna and mki67; Fig. 2D). Cluster JM5 contained too few cells to be definitively assigned. The remaining three clusters (JM1, JM0, JM4) had microglial identity but distinct gene expression profiles. 
Next, we examined whether these subsets persist into adulthood and mapped their regional distribution in the brain by co-clustering our juvenile data with adult brain microglia that were sorted and sequenced in parallel. The Harmony $R$ package ${ }^{42}$ was used to integrate the two data sets and compensate for sources of technical variation. Unbiased clustering and differential expression analysis revealed six groups that were conserved between juveniles and adults (Fig. 2E; Table S3) as well as a distinct adult-enriched microglial cluster (A4; Fig. 2F). Notably, adult cluster A3 mapped to juvenile cluster JM4 (conserved genes included cebpb, c1qa, c1qb, and $c 1 q c$ ), whereas adult cluster A1 mapped to juvenile cluster JM1 (apoeb and ctsba). A proliferative cluster was still present (A2; pcna, tubb2b, and mki67), as well as two distinct macrophage clusters (A5 and A6) corresponding to juvenile cluster JM3 (ccl19a, siglec15l, and cmklr1). Interestingly, the novel adult-enriched cluster A4 was distinctly enriched in inflammasome genes, suggesting a microglial subset poised for inflammasome activation (caspb, fads2, and alox5ap; Fig. 2G). Taken together, these data reveal myeloid cell heterogeneity in the juvenile zebrafish brain that persists in the adult.

\section{Region-specific transcriptional signatures of juvenile zebrafish myeloid cells}

Previous zebrafish transcriptomes using the Tg(mpeg:EGFP) transgenic line and bulk sequencing approaches have suggested regional transcriptional heterogeneity within the zebrafish brain ${ }^{31}$. To better compare our single cell data with the existing literature, we isolated mpeg:EGFP+ myeloid cells from OT, midbrain, and hindbrain at $28 \mathrm{dpf}$ by flow cytometry and performed bulk RNA sequencing (Fig. 3A, Fig. S3A-B, Table S4). The resulting transcriptome was highly enriched for microglial specific genes (csf1rb, c1qa, and p2ry12), as well as some macrophage markers (siglec15l, spock3). This was a pure myeloid population with no evidence of neuronal, astrocyte, or oligodendrocyte genes (Fig. S3C). Principal component analysis revealed that $60 \%$ of gene variance was driven by brain region (PC1; Fig. 3B), and differential expression analysis of these subsets $(p<0.05)$ identified region specific gene expression signatures (Fig. $3 \mathbf{C})$. In particular, complement and antigen presentation genes were enriched in the hindbrain (c1qa, $c 1 q c, c d 74 a$, and $m h c 2 d a b)$, whereas lysosome-associated genes were highly enriched in the OT (apoeb, ctsba, ctsc, and ctsla). The midbrain was intermediate and did not clearly segregate with either phenotype.

We confirmed region-specific expression for two candidate genes from this profile via in situ hybridization (ISH). The HB enriched gene cd74a was expressed in over $90 \%$ of hindbrain microglia (mpeg $+4 c 4+$ ) and $30 \%$ of OT microglia, and intermediate in the midbrain (Fig. 3D-E). In contrast, the OT-enriched gene ctsba was highly enriched in neurogenic areas and highest in the OT compared to midbrain and hindbrain $(72 \%, 33 \%, 19 \%$, respectively; Fig. 3F-G). These data reveal brain-region specific transcriptional signatures that can be identified in situ. However, they also suggest that these differences may be partly driven by varying proportions of phenotypically distinct microglia as well as resident macrophages that are better discriminated at the single-cell level of resolution.

\section{Region-enriched functional microglial subsets in zebrafish hindbrain and optic tectum}

We next used our region-specific transcriptional signatures to map our single cell data, with the goal of localizing functional subsets in situ (Fig. 4A). To do this, we calculated an "eigengene" composed of the top differentially expressed genes in the mpeg+ population from each region. Overlay of these region-defining eigengenes onto our UMAP plot revealed substantial enrichment of the OT signature in the juvenile cluster JM1, whereas the hindbrain eigengene was enriched in 
JM4 and the macrophage cluster JM3 (Fig. 4B-C). The midbrain regional signature was indeterminate and aligned only with the macrophage cluster, consistent with our finding of substantially more macrophages in this region (Fig. S1D). Cluster JM0 was not enriched in any regional signature, suggesting that it may represent a common microglial population, or a subset not represented in our bulk sequencing. Since the OT-enriched cluster JM1 and the hindbrainenriched cluster JM4 were distinct in both bulk and single-cell sequencing and did not contain contaminating macrophages, we directly compared the transcriptomic profile of these subsets (Fig. 4D-E).

Genes that defined the hindbrain-enriched cluster (JM4) included c1qa, the initiating protein in the complement cascade, a known regulator of synaptic engulfment expressed in rodent microglia 43,44 . Another was cebpb, a transcription factor that promotes microglial homeostasis in neurodegenerative disease ${ }^{45}$. Other genes included the hindbrain region defining gene $c d 74 b$, which encodes the invariant chain of major histocompatibility complex class II, and grn1, which encodes progranulin and interacts with $\mathrm{C} 1 \mathrm{q}$ to regulate synaptic pruning ${ }^{46}$. We also identified several putative functional genes not previously studied such as the lysozyme gene lyg/1. Gene ontology analysis suggested that the preferentially expressed genes are involved in protein production and metabolism, including translational elongation, negative regulation of proteolysis, and protein folding (Fig. 4D-E). In contrast OT-enriched cluster JM1 was highly enriched for lysosomal activity, including multiple genes for lysosomal proteases including cathepsins (ctsba, ctsz, ctsla, ctsc, and ctsd) as well as higher levels of apoeb and Igals9/1. Thus, functional subsets identified by scRNA seq correlate with the differentially enriched populations of synapseassociated and neurogenic associated microglia that we identified in situ.

228

229

230

231

232

233

234

235

236

237

238

239

240

241

242

243

244

245

246

247

248

249

250

251

252

253

We hypothesized that hindbrain-enriched cluster JM4 represents a synapse-associated microglia subset whereas JM1 preferentially associates with neurogenic regions. To identify our putative OT cathepsin-rich cluster (JM1) in situ, we quantified functional cathepsin activity with the biomarker Prosense 680, which becomes fluorescent after proteolytic cleavage by cathepsins with a preference for cathepsin $B$ and $L^{47}$. Microglial cathepsin activity (mpeg+prosense + ) was highly enriched in the OT, where it was mostly detected in ameboid microglia around neurogenic regions (Fig. 4F-G), closely matching the expression of ctsba by in situ hybridization (Fig. 3F-G). Consistent with this, tectal microglia engulfed substantially more cell corpses, as quantified by uptake of a neuronal-soma enriched DsRed $\operatorname{Tg}(N B T: D s R e d)$ compared to the hindbrain (Figure S4A-B). Next, we quantified synapse engulfment by 3D reconstruction of mpeg-EGFP positive cells with the synaptic marker SV2. We found that hindbrain microglia engulfed significantly more SV2 than OT microglia, consistent with enrichment of the JM4 cluster in hindbrain (Fig. 4H-I). Importantly, stratifying this data by morphology revealed that ramified microglia (sphericity <0.6) engulfed more SV2 regardless of brain region, although they were markedly more abundant in the hindbrain. These data suggest functional subsets whose abundance differs between brain regions, rather than strictly region-specific functions. Taken together, we conclude that our cathepsin-enriched scRNA Seq cluster JM1 corresponds to cell corpse-engulfing microglia highly enriched in neurogenic regions, whereas complement-expressing cluster JM4 identified a synapse-associated subset.

\section{Discussion}

Here we define the regional localization and molecular signatures of two distinct microglial phagocytic states in the developing zebrafish, including a novel subset of synapse-associated 
microglia (SAMs) abundant in the hindbrain (Fig. 5 A, B). This functionally annotated single cell dataset is an opportunity to investigate fundamental questions related to interactions between microglia and synapses. For example, microglia both engulf synapses and promote synapse formation via modifying the extracellular matrix and other mechanisms ${ }^{1,48,49}$. The molecular mechanisms that regulate these different states are not well understood. The impact of neuronal activity on microglial function is also a major area of interest: microglial synapse engulfment has been proposed to be activity-dependent, and in both fish and rodents microglial contact can acutely regulate neuronal activity ${ }^{50,51}$. However, observing these processes in the intact developing brain is extremely challenging in rodents, and is a major strength of the zebrafish model. This molecularly defined population of synapse-associated microglia in zebrafish hindbrain provide an opportunity to temporally define and genetically manipulate these microglial subsets in physiological and in disease contexts.

Importantly, we show that both SAMs and NAMs are phagocytic in vivo, but in different contexts and via distinct molecular mechanisms. Our observations regarding NAMs are consistent with several recent publications: deficits in lysosome function result in defective cell corpse phagocytosis and dysmorphic 'bubble microglia' in the OT ${ }^{20}$. Another study used bulk RNA sequencing to identify $c c / 34$ as a marker of these ameboid phagocytic microglia in the adult zebrafish, a marker which is also significantly enriched in our NAM subset ${ }^{31}$. Our study defines the transcriptome of this subset at single cell resolution, and establishes an in vivo assay to track lysosomal function in this population. More importantly, we have identified novel genes associated with a microglial population that is not well-described in the zebrafish -- synapse-associated microglia. The initiating components of the classical complement cascade (c1qa and c1qc) are top differentially expressed genes in our SAM subset. C1q is also microglial-encoded in the murine brain and in multiple vertebrate species including zebrafish ${ }^{33,43}$ and promotes developmental synaptic engulfment ${ }^{52}$. Other top candidates, including the transcription factor cebpb and major histocompatibility complex, class II (cd74a \& cd74b), have been implicated in microglial function in rodent models of Alzheimer's disease ${ }^{45,53}$. These data suggest that the SAM profile identifies a core microglial program conserved across species, and raise the question of how other gene candidates, including $l y g / 1, g r n 1, f g / 2 a$ and others, are regulating microglialsynaptic interactions.

Our data highlight evolutionarily conserved features of zebrafish microglia as well as unique strengths of the zebrafish model. It is striking that developmental microglial heterogeneity in the fish is largely conserved into adulthood, in contrast to generally diminishing amounts of diversity in adult rodents ${ }^{3,4}$. This may reflect the fact that fish continue to grow in size throughout life, adding new neurons and new synaptic connections. This suggests that the time window for studying microglial roles in circuit remodeling may be much broader than in mammals, and that mechanisms may exist to maintain synaptic plasticity throughout life. Future studies focusing on microglial-synapse interactions in the zebrafish hindbrain can take advantage of co-existing populations of cell-corpse engulfing and synapse associated microglia to further define the differences between these subsets. Our data also suggest that live-imaging of microglial-synapse interactions may help to answer key questions about how and why microglia interact with synapses. Finally, the ability to do high throughput screening in zebrafish raises the possibility that this model could be used to define new therapeutic targets, in neurodevelopmental diseases linked to immune dysfunction including autism spectrum disorder, epilepsy, and schizophrenia.

300 
Silva et al.

\section{Acknowledgements:}

We are grateful to Marci Rosenberg, Haruna Nakajo, and members of the Molofsky Lab for helpful comments on the manuscript, to Ari Molofsky, Tom Nowakowski and Galina Schmunk for their expert advice, and to Brian Black, Gary Moulder and Louie Ramos for the support of the CVRI Zebrafish Core facility. Thanks to Francesca Peri for the Tg(mpeg:EGFP-caax), David Traver for the $T g(C d 45: D s R e d)$ fish, and Roland Wu for the Tg(mpeg:EGFP) fish, and to the UCSF Laboratory for Cell Analysis and Dr. Eric Chow at Center for Advanced Technologies for technical contributions. Funding: A.V.M is supported by the Pew Charitable Trusts, NIMH (R01MH119349 and DP2MH116507), and the Burroughs Welcome Fund. N.J.S. is supported by UCSF-IRACDA (K12) fellowship. L.C.D. received support from the Matilda Edlund Scholarship and the Genentech Fellowship.

\section{Author contributions:}

Conceptualization: N.J.S, A.V.M, I.D.V., and L.C.D.; Methodology, N.J.S., I.D.V, and L.C.D; Investigation, N.J.S, I.D.V, L.C.D, and N.C.H; Writing - Original Draft, N.J.S, L.C.D., and A.V.M.; Writing - Review \& Editing, all co-authors; Funding Acquisition, A.V.M. and N.J.S. Resources, A.V.M.; Supervision, A.V.M. and I.D.V

Declaration of interests: The authors declare no competing interests.

Data and materials availability: Supplement contains additional data. All data needed to evaluate the conclusions in the paper are present in the paper or the Supplementary Materials. Single cell RNA sequencing and bulk RNA sequencing data were deposited in NCBl's Gene Expression Omnibus (Barrett et al., 2013) under accession numbers GSE164772 and GSE164771, respectively. 
Figures and Figure legends:

A

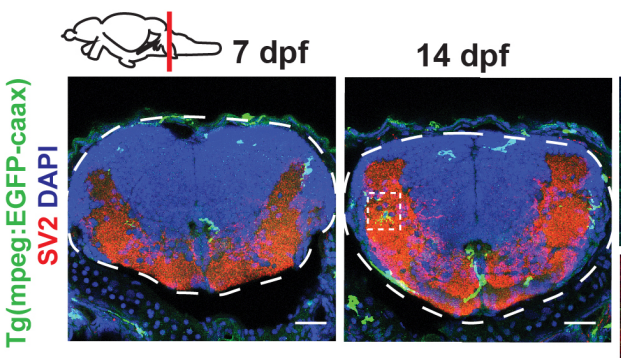

C

$\mathrm{Tg}$ (mpeg:EGFP-caax) SV2 DAPI

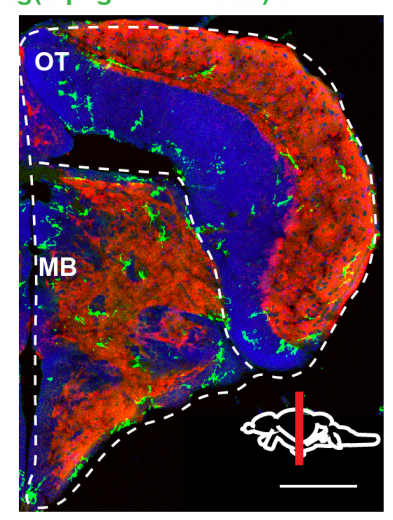

G

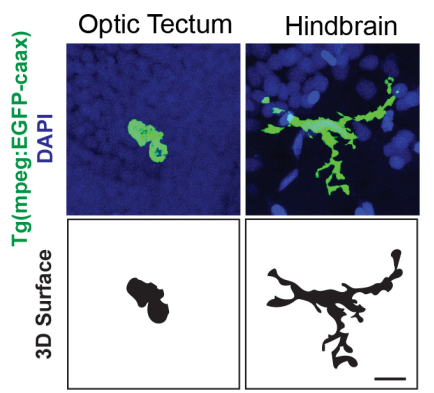

B
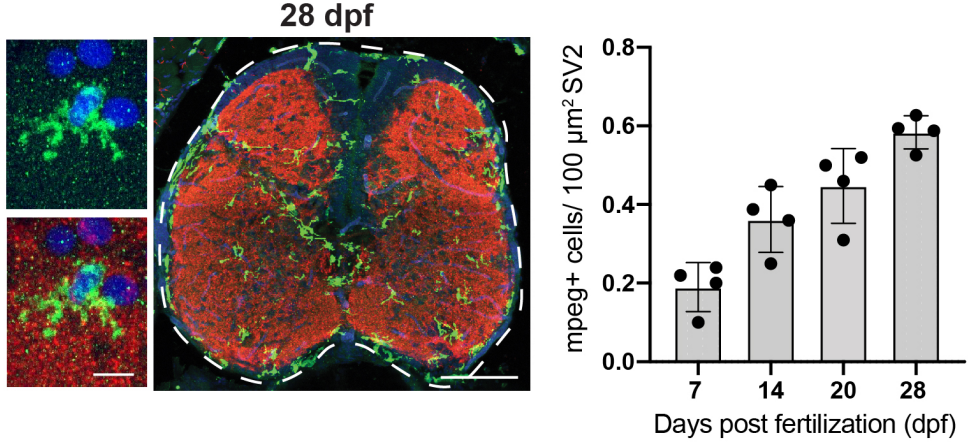

E

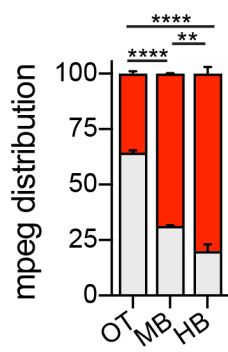

घ Within SV2

口 Outside SV2

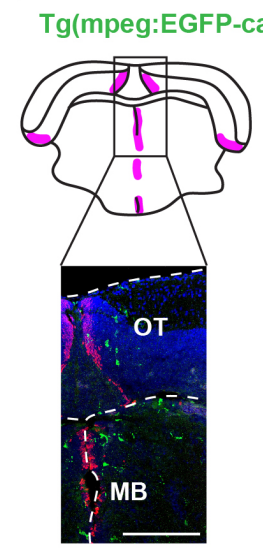

$\mathbf{F}$

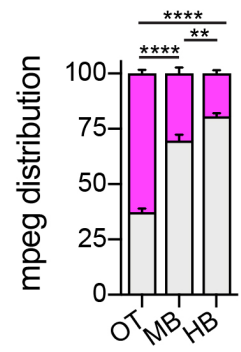

口 Within BrdU

$\square$ Outside BrdU

Figure 1. Phenotypically distinct populations of microglia in synaptic and neurogenic regions of zebrafish brain.

(A) Representative images of microglia (mpeg:EGFP) and synapses (SV2 antibody stain) in developing zebrafish hindbrain. Inset: representative synapse-embedded microglia at $14 \mathrm{dpf}$. Scales: $7-14 \mathrm{dpf}, 20 \mu \mathrm{m} ; 28 \mathrm{dpf}, 50 \mu \mathrm{m}$; inset, $5 \mu \mathrm{m}$.

(B) Quantification of mpeg:EGFP+ cells per $100 \mu \mathrm{m}^{2}$ of SV2+ synaptic area at 7,14, and 28 days post fertilization. Dots represent individual fish, data are mean \pm SD. 


\section{Silva et al.}

340 (C-D) Representative images and quantification of the proportion of mpeg:EGFP+ cells in 341 synaptic (SV2+; red) vs. cellular (DAPI+/SV2-; grey) areas. Distribution quantified within each 342 brain region as outlined (dotted lines): midbrain (MB), optic tectum (OT), and hindbrain (HB, see 343 panel $1 \mathrm{~A}$ ). Mean \pm SEM from 4 fish. Scale: $50 \mu \mathrm{m}$.

344 (E-F) Representative images and quantification of the proportion of mpeg:EGFP+ cells within 20 $345 \mu \mathrm{m}$ of BrdU+ neurogenic regions (purple) vs. outside neurogenic regions (grey). Mean \pm SEM from 3463 fish. Scale: $50 \mu \mathrm{m}$.

347 (G) Representative images of mpeg:EGFP+ microglia and thresholded maximal projections in OT 348 and $\mathrm{HB}$ at $28 \mathrm{dpf}$. Scale: $5 \mu \mathrm{m}$.

349 (H) Quantification of microglial sphericity (Scale 0-1, 1=most spherical/ameboid), from images

Statistics: Two way ANOVA with Tukey's post-hoc comparison (D,F), two-tailed unpaired t-test $(\mathrm{H}, \mathrm{K}) .{ }^{*} \mathrm{p}<0.05,{ }^{* *} \mathrm{p}<0.01,{ }^{* * *} \mathrm{p}<0.001,{ }^{* * * *} \mathrm{p}, 0.0001$

\section{See also Fig S1.}


A

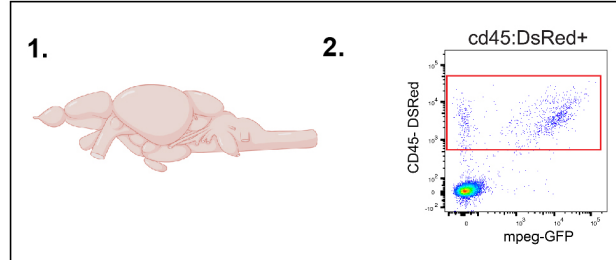

B
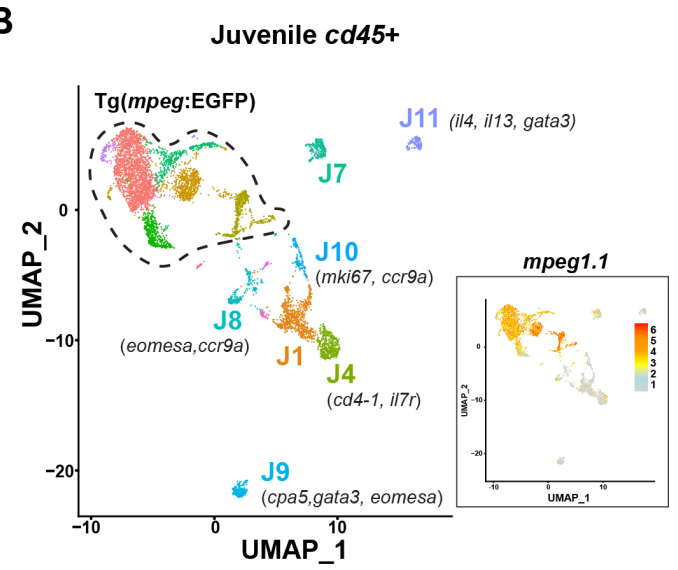

D
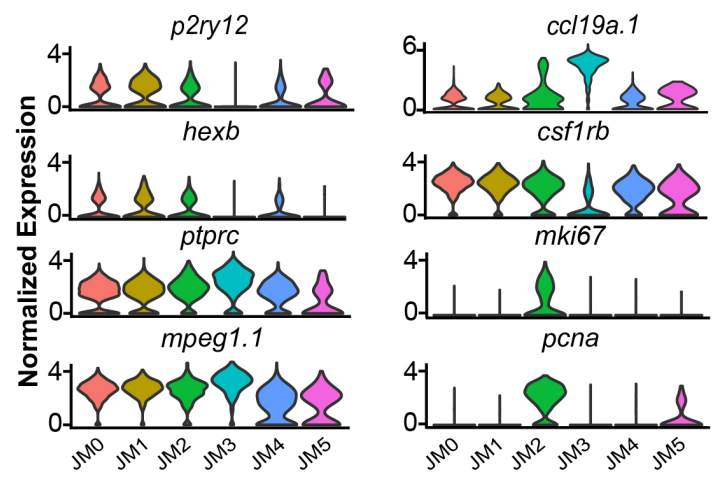

3.

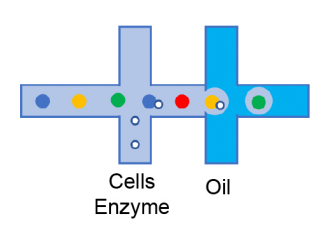

C

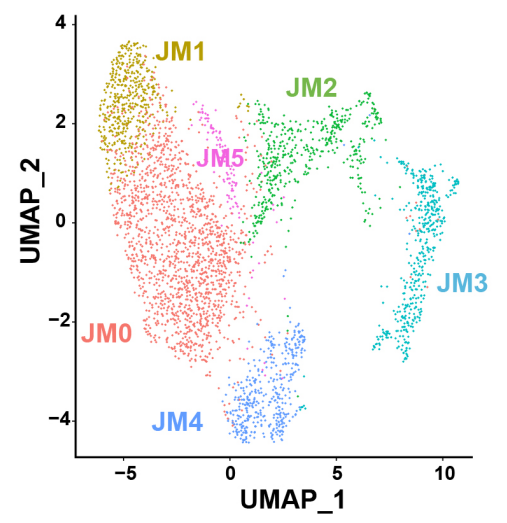

E
4.

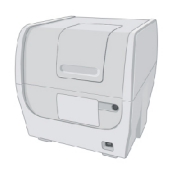

F

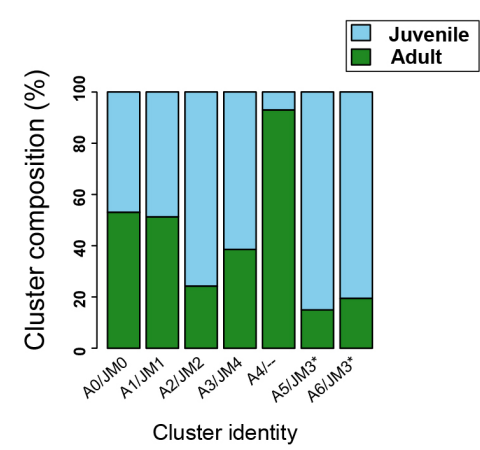

G

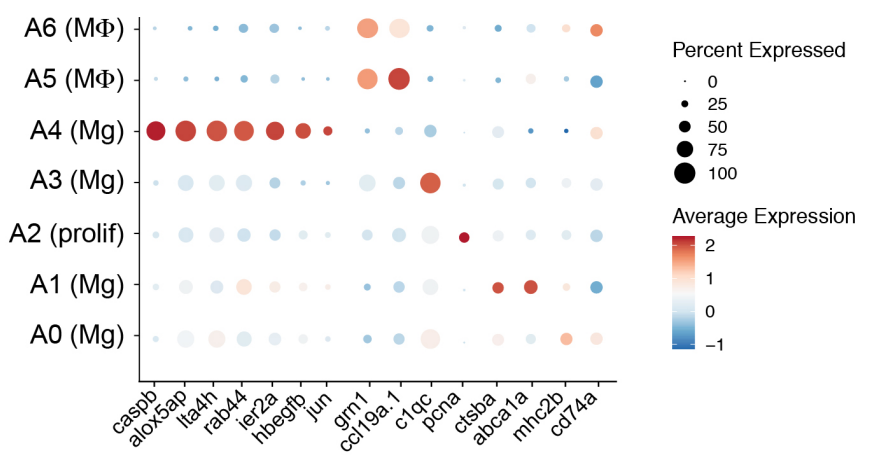

Figure 2. Molecularity distinct subsets of microglia identified at single cell resolution during brain development and in adulthood.

(A) Schematic diagram of whole brain scRNA -sequencing pipeline.

(B) Unsupervised clustering of 6,666 juvenile ptprc (CD45)+ cells. Inset: feature plot of mpeg1.1 expression. $\mathrm{n}=10$ fish in two independent replicates. 


\section{Silva et al.}

364 (C) Subclustering of 3,539 mpeg1.1+ cells in C. Inset: cluster dendrogram.

365 (D) Violin plots for select marker genes across juvenile clusters from C (JM1-5), including 366 microglia (p2ry12, hexb, and csf1rb), pan-myeloid (mpeg1.1), pan-hematopoietic (ptprc/cd45), 367 macrophage (ccl19a.1) and proliferative (mki67 and pcna).

368 (E) UMAP plots showing co-clustering analysis of juvenile cells in $C$ and 2,080 adult myeloid 369 (mpeg1.1+) cells. Conserved macrophage and proliferative clusters indicated. Dotted line outlines 370 remaining 'homeostatic' microglial clusters.

371 (F) Percent of each cluster shown in E consisting of Juvenile (blue) vs. Adult (green) derived cells. 372 Normalized within groups to account for overall cell number difference between ages.

373 (G) Dot plot highlighting select up- and downregulated genes within adult clustering in $\mathrm{E}$.

\section{See also Fig S2.}


A

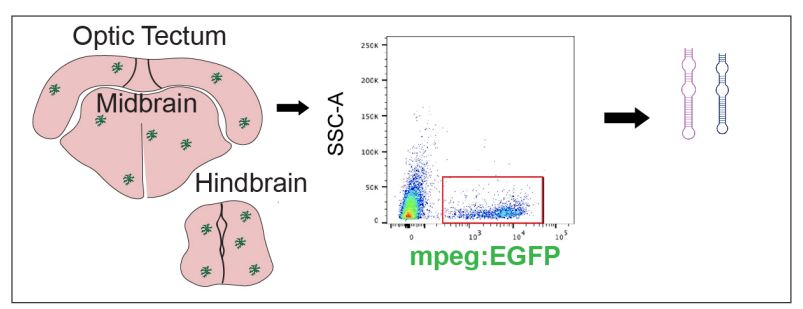

B

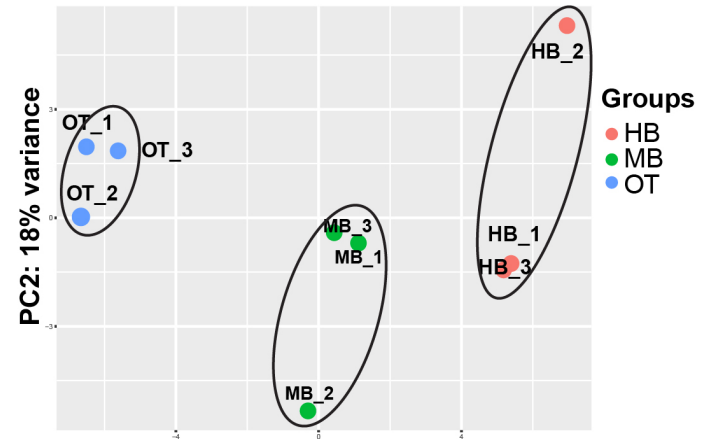

PC1: $60 \%$ variance

D

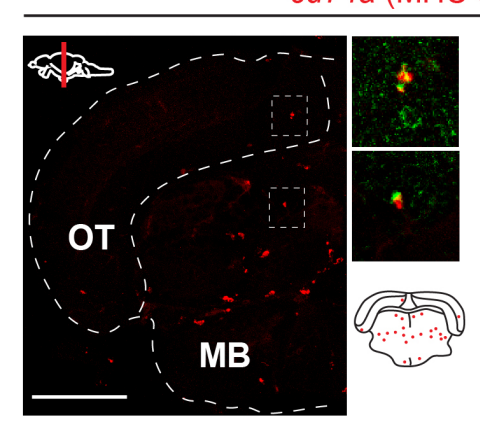

F
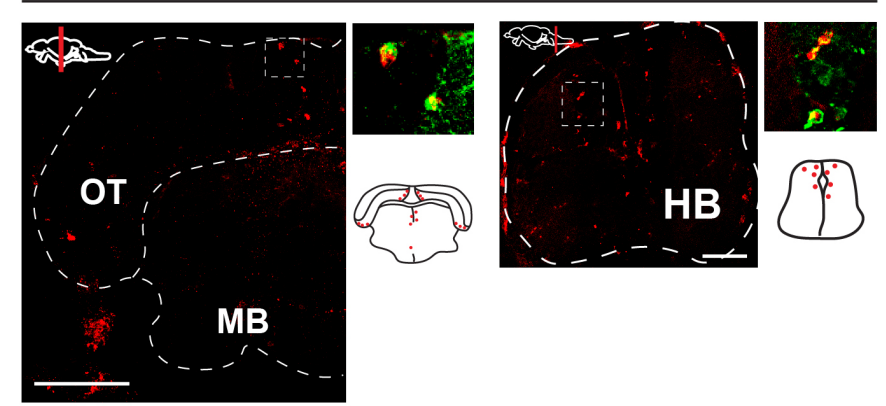

C

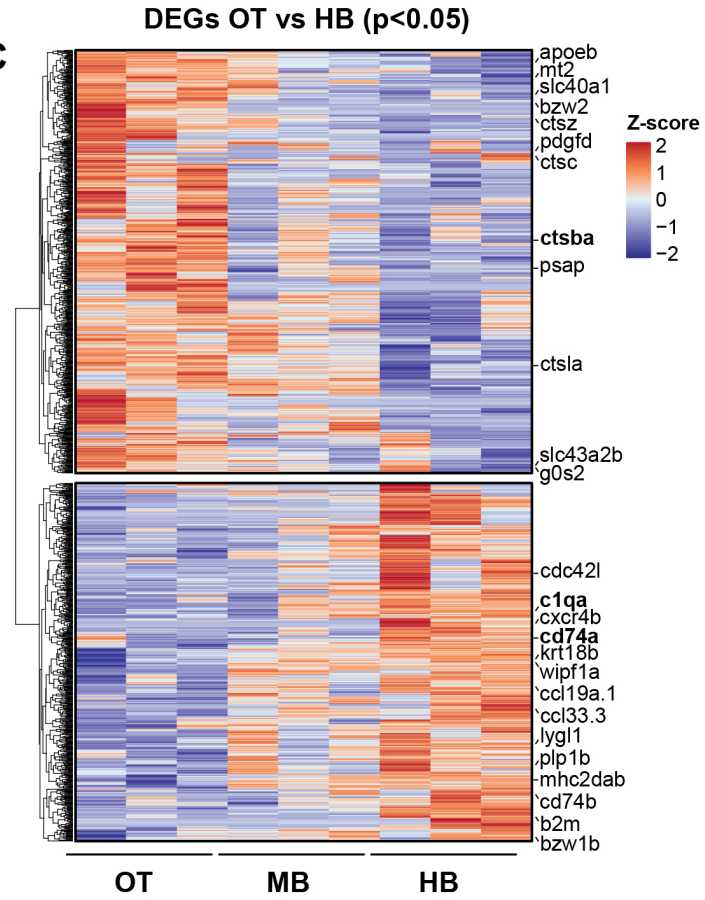

E

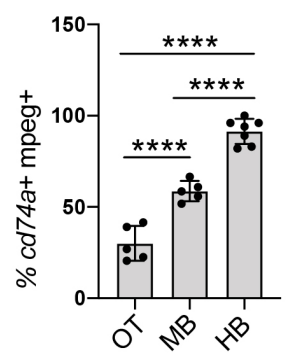

G

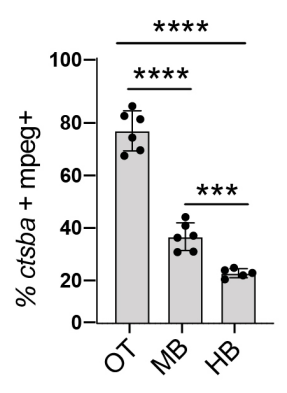

(A) Schematic of region-specific bulk-sequencing approach using mpeg:EGFP.

(B) Principal component analysis (PCA) plot of top 500 most variable genes for OT, MB, HB

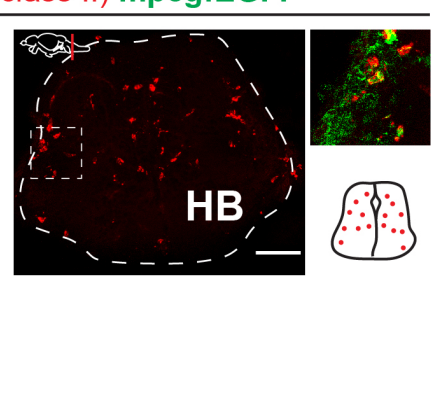
regions (dots= independent replicates from 10 pooled fish/replicate). 


\section{Silva et al.}

384 (C) Heatmap of differentially expressed genes (DEGs) from mpeg:EGFP positive cells across 385 brain regions (Adjusted $p$-value <0.05). Selected top differentially expressed genes from each 386 cluster highlighted.

387 (D-E) Representative images and quantification of cd74a expression across brain regions by in 388 situ hybridization (ISH), co-localized with mpeg-GFP. Quantification shows percentage of cd74 + 389 mpeg+ microglia within each brain region. Scale: $50 \mu \mathrm{m}$.

390 (F-G) Representative images and quantification of ctsba expression by ISH colocalized with 391 mpeg-GFP. Quantification shows percentage ctsba+ mpeg+ microglia within each brain region. 392 Scale: $50 \mu \mathrm{m}$.

394 Statistics: In E,G, One way ANOVA with Tukey's post hoc test. Mean $\pm S D$, dots=individual fish. $395{ }^{*} p<0.05,{ }^{* *} p<0.01,{ }^{* * *} p<0.001,{ }^{* * *} p<0.0001$.

\section{See also Fig. S3.}


A

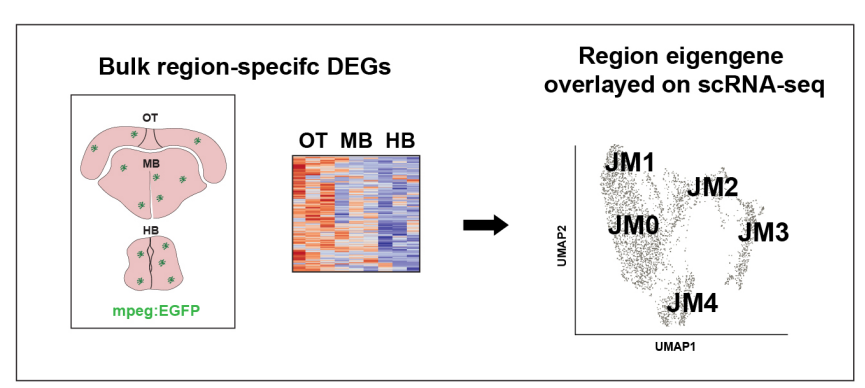

C

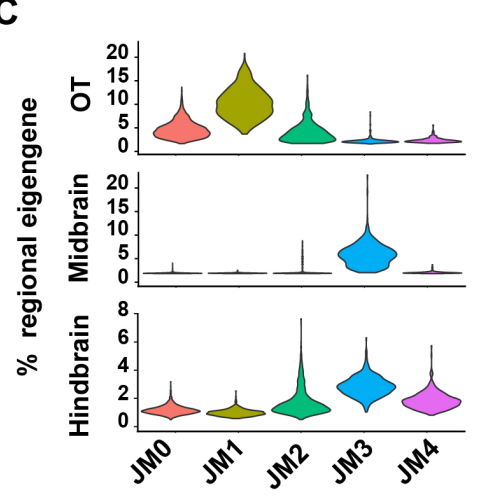

D

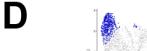

JM1 vs. JM4

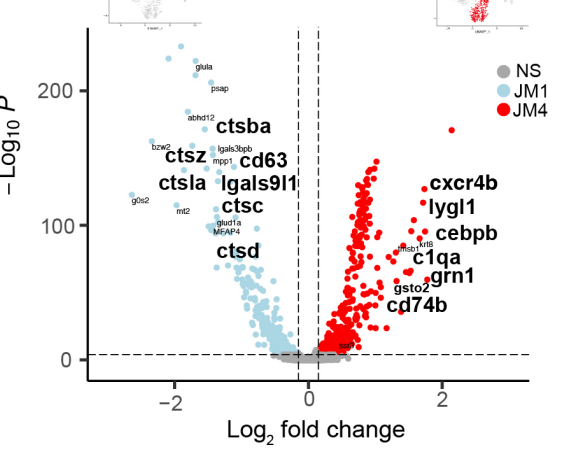

$\mathbf{F}$

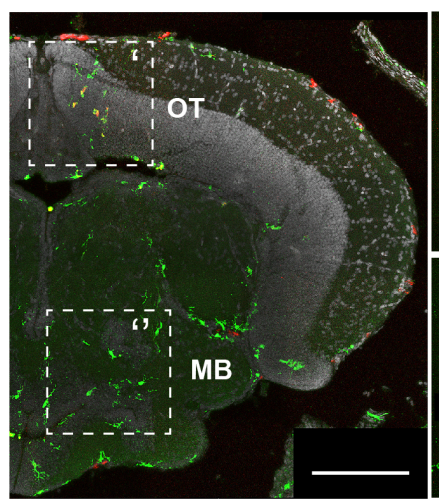

$\mathbf{H}$

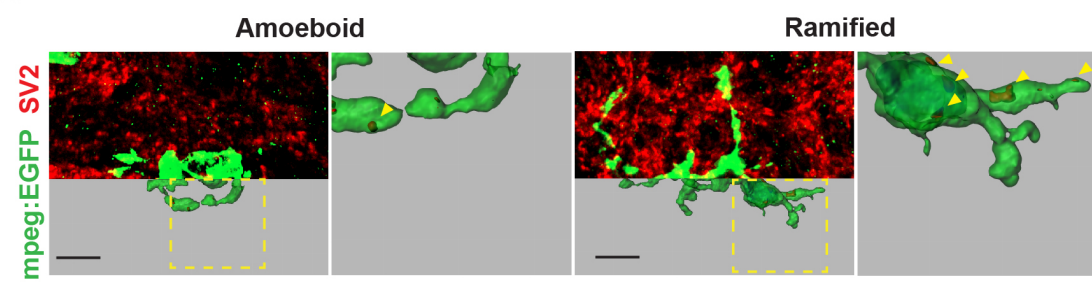

B

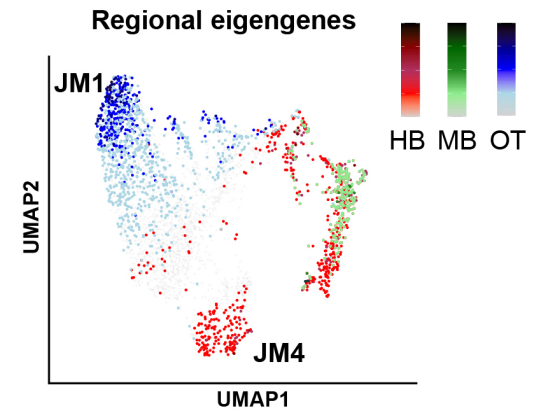

E

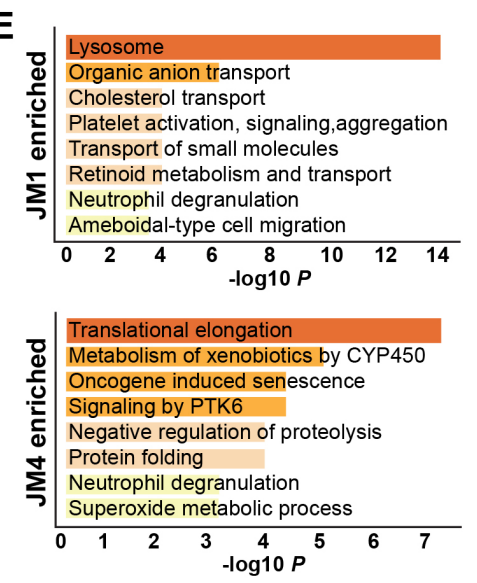

G

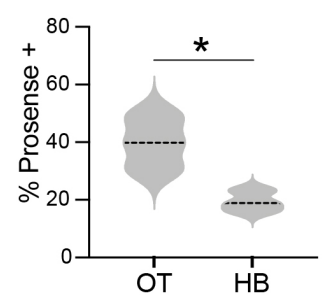

- Amoeboid
Ramified

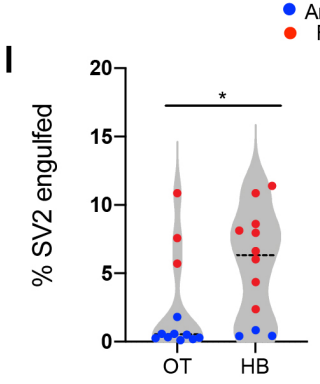

Figure 4. Region-enriched functional microglial subsets in zebrafish hindbrain and optic tectum.

(A) Schematic of analysis pipeline overlaying brain-region defining genes identified with bulk sequencing onto mpeg+ scRNA seq clusters. 
(B) Feature plots of region-defining eigengenes from hindbrain (HB; red), midbrain (MB; green) and optic tectum (OT; blue) overlaid on mpeg+ UMAP, highlighting microglial clusters JM1 and

405 JM4.

406 (C) Violin plots of regional eigengene distributions, related to $B$.

407 (D) Volcano plot of differentially expressed genes between clusters JM1 (OT-enriched; blue) and 408 JM4 (hindbrain-enriched; red). Thresholds represented by dotted lines were set to adjusted $p$ 409 value $<10^{-8}$, log fold change $>0.2$.

410 (E) Top GO terms from differentially expressed genes in $D$.

411 (F) Representative images of cathepsin-cleaved Prosense 680 colocalized with mpeg:EGFP in indicated brain regions. Scale: $100 \mu \mathrm{m}$.

413 (G) Quantification of percent microglia containing cleaved Prosense680 in OT and HB. Unpaired t-tests. Mean \pm SD. ${ }^{*} p<0.05$. OT (Optic Tectum) and HB (Hindbrain).

(H) Representative images and 3D reconstructions of mpeg:EGFP+ microglia with engulfed SV2 protein. Insets: close-up of reconstructions (arrowheads:SV2 content). Scale bar $5 \mu \mathrm{m}$. (I) Quantification of percent microglial volume containing SV2 in randomly selected microglia from OT and HB. Post hoc analyses show morphology assignment as ramified (red, sphericity<0.6) vs. amoeboid (blue, sphericity $>0.6$ ). Unpaired t-tests within regions, ${ }^{* * *} \mathrm{p}<0.001$, dots $=$ mean value per fish from 3 microglia per fish.

Statistics: Two-tailed unpaired t-test $(G, I) .{ }^{*} p<0.05,{ }^{* *} p<0.01,{ }^{* * *} p<0.001,{ }^{* * * *} p, 0.0001$ 
Silva et al.

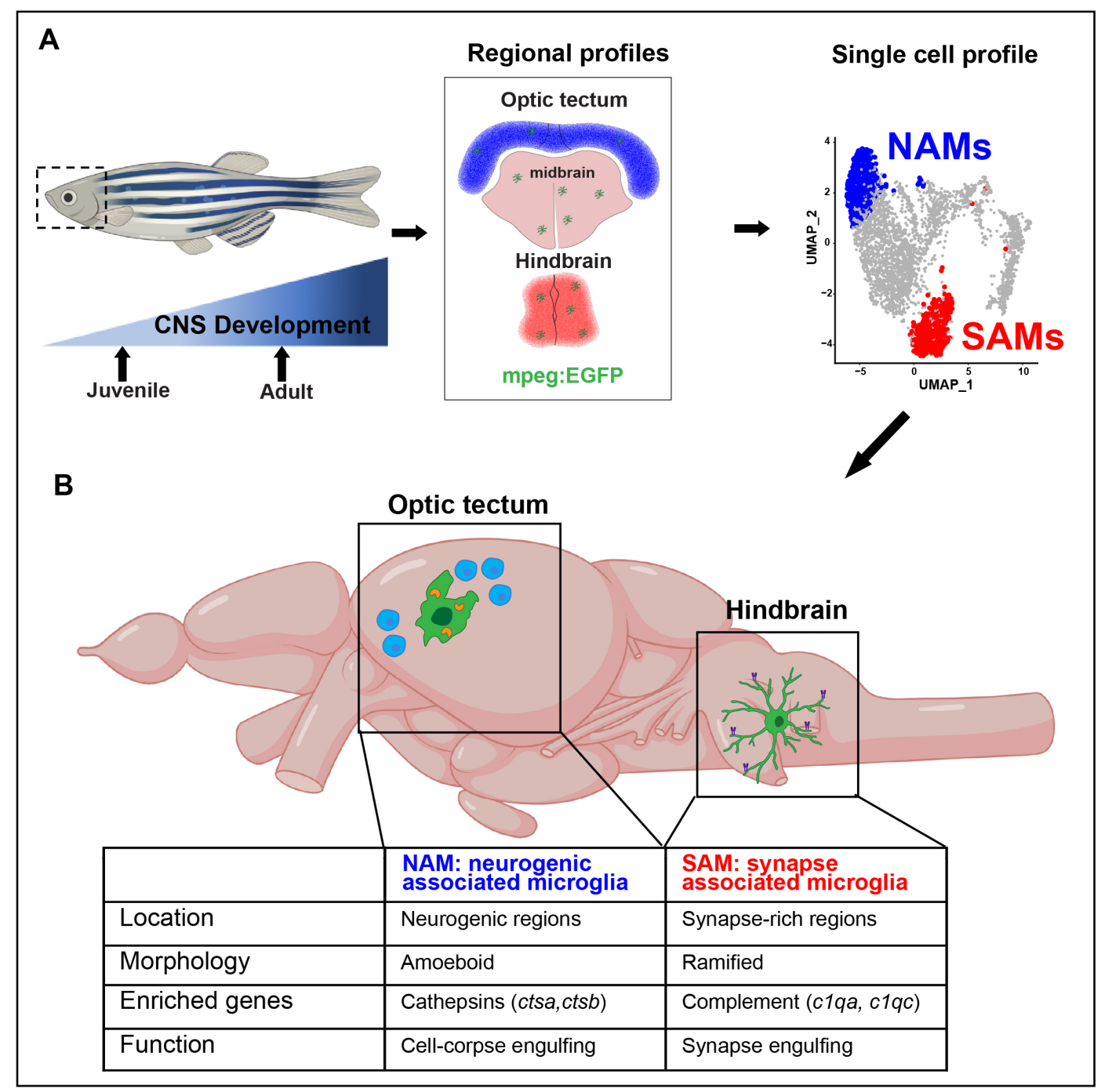

Figure 5. Summary diagram of the identified neurogenic-associated microglia (NAMs) and synapse-associated microglia (SAMs) in the developing zebrafish brain.

(A) Schematic of analysis pipeline overlaying brain-region defining genes identified with bulk sequencing onto mpeg+ scRNA seq clusters identifies NAMs and SAMs.

(B) Unique features of NAMs and SAMs in the developing juvenile brain.

Supplemental information:

Figs S1-S4

Tables S1-S3

Methods 


\section{mpeg:EGFP-caax (Macrophage) 4C4 (Microglia) DAPI at 28 days post fertilization (dpf)}

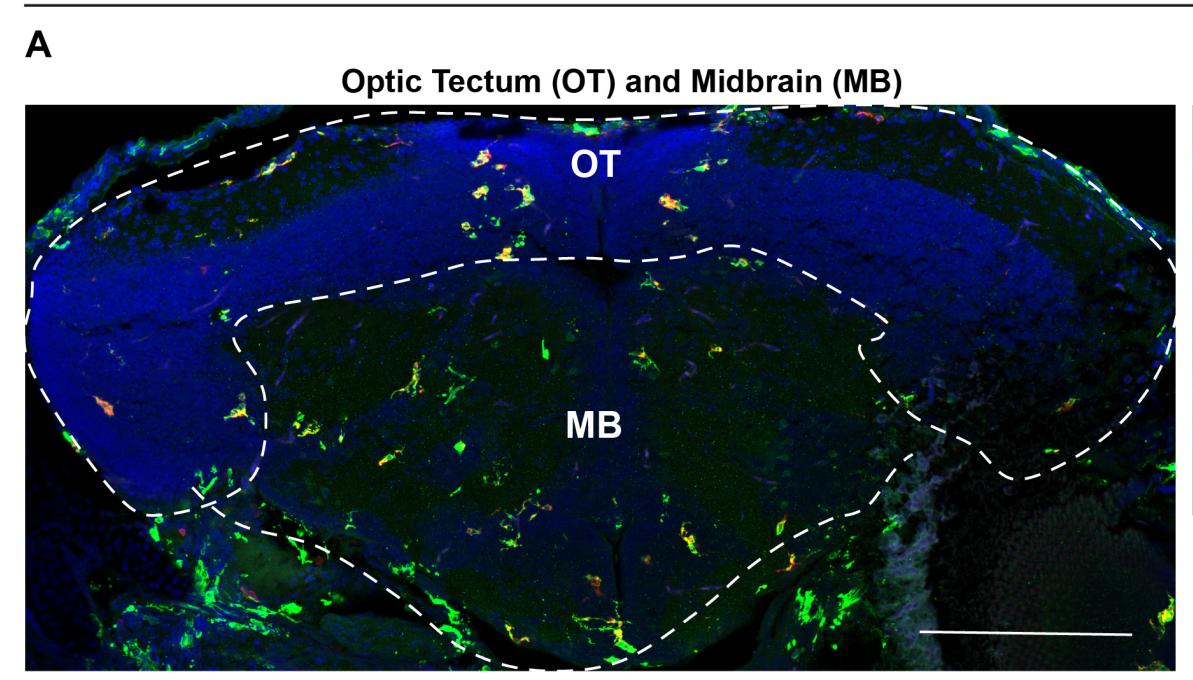

B

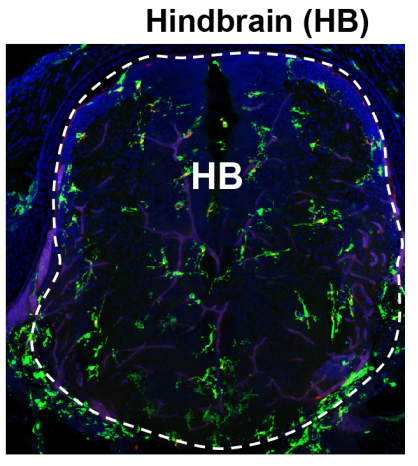

C

D
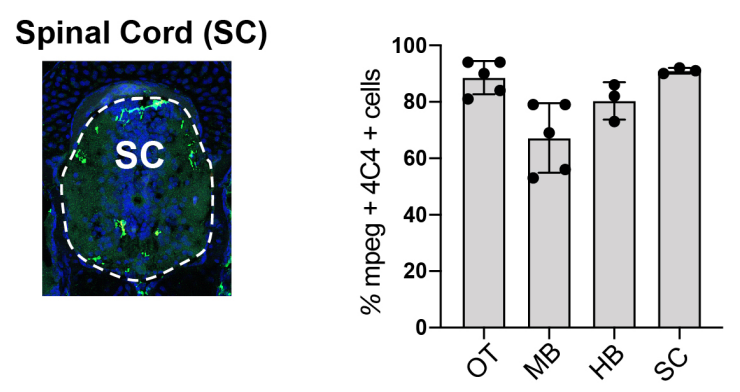

442

Figure S1. Macrophages and microglia in OT, MB, and HB regions at $28 \mathrm{dpf}$, supplemental to Figure 1.

(A-C) Representative images of brain regions using the pan-myeloid reporter line mpeg:EGFP and the microglial-specific antibody $4 \mathrm{C} 4$ at 28 days post fertilization (dpf).
(D) Quantification of percent of mpeg:EGFP+ population also expressing 4C4.

Scale: $100 \mu \mathrm{m}$. Optic tectum (OT), midbrain (MB), and hindbrain (HB). 
Silva et al.

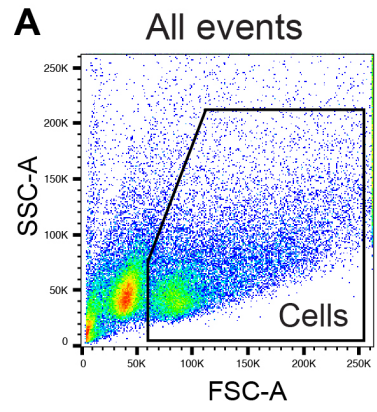

B

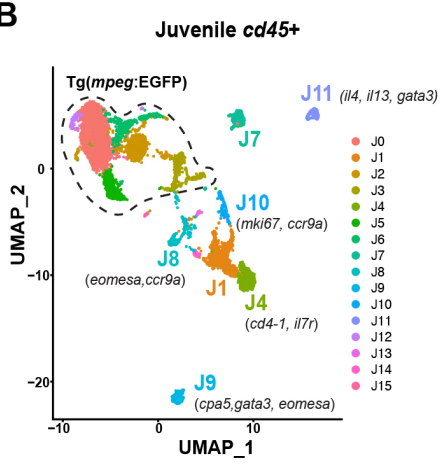

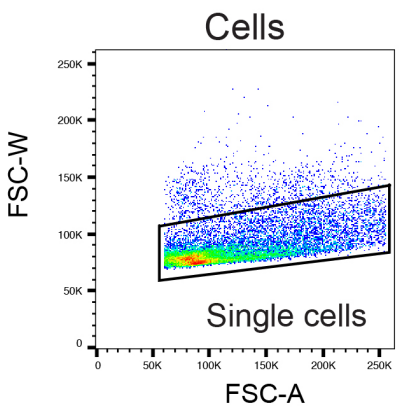

C

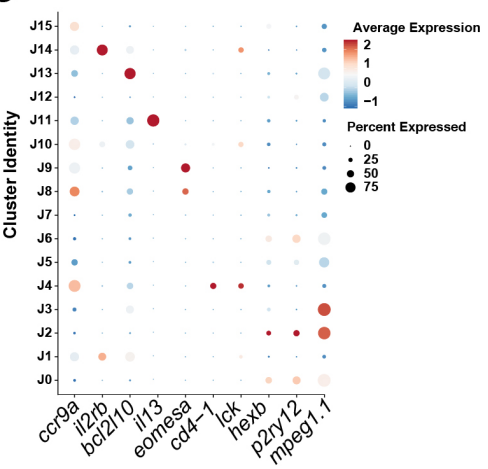

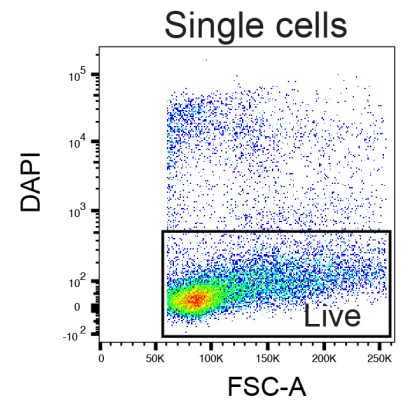

D

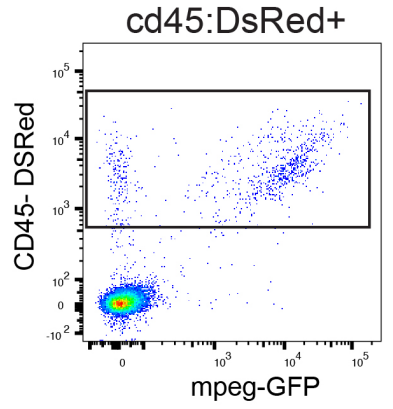

E

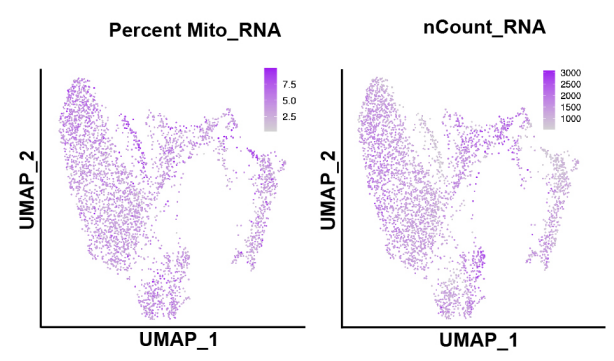

$\mathbf{F}$

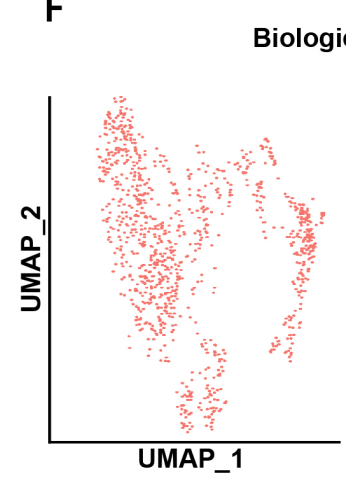

Biological replicates

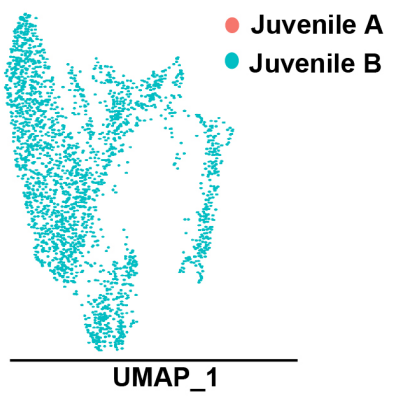

G

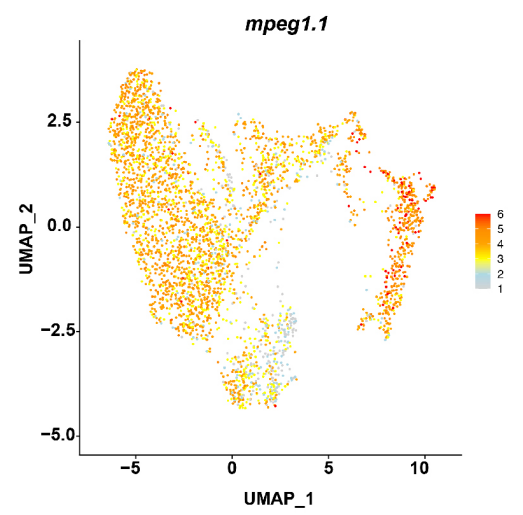

456

457

458

459

460

461

462

463

464

465

466

467

Figure S2. Gating hierarchy and quality control of single cell sequencing data, supplemental to Figure 2.

(A) Gating strategy to isolate $c d 45$ positive cells for single-cell RNA sequencing.

(B) Unsupervised clustering of juvenile cd45 positive cells, replicated from Fig. 2B for reference.

(C) Select enriched genes in cd45+mpeg-immune cell subsets.

(D-E) Feature plot showing distribution of mitochondrial RNA content and genes recovered (nCount) per cell.

(F) Comparison of independent biological replicates of mpeg+ cells pooled in Fig. $2 \mathrm{C}$.

(G) UMAP plot highlighting levels of mpeg1.1 expression the mpeg1.1+ population from 2C. 
Silva et al.

A

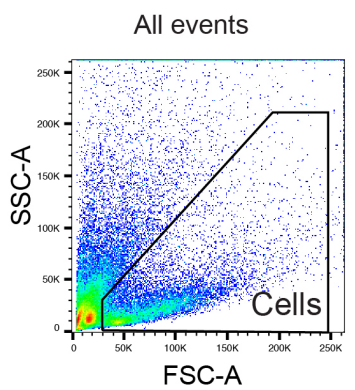

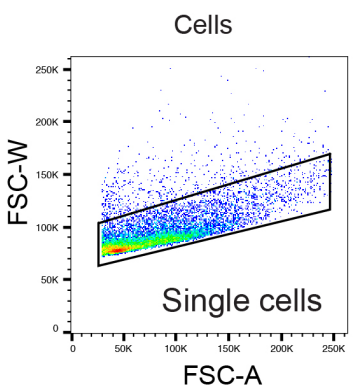
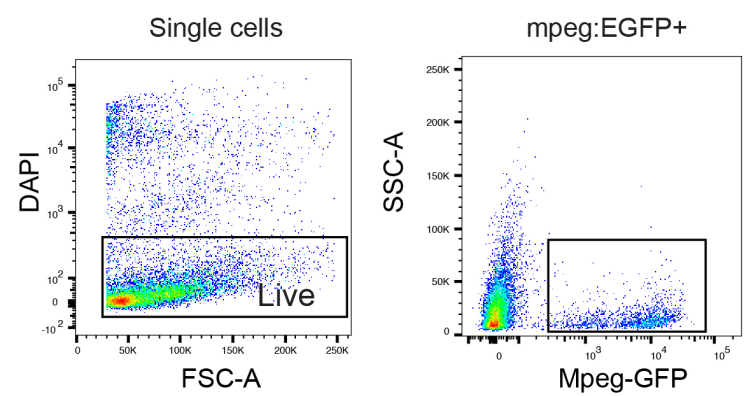

B

Cell type specific genes

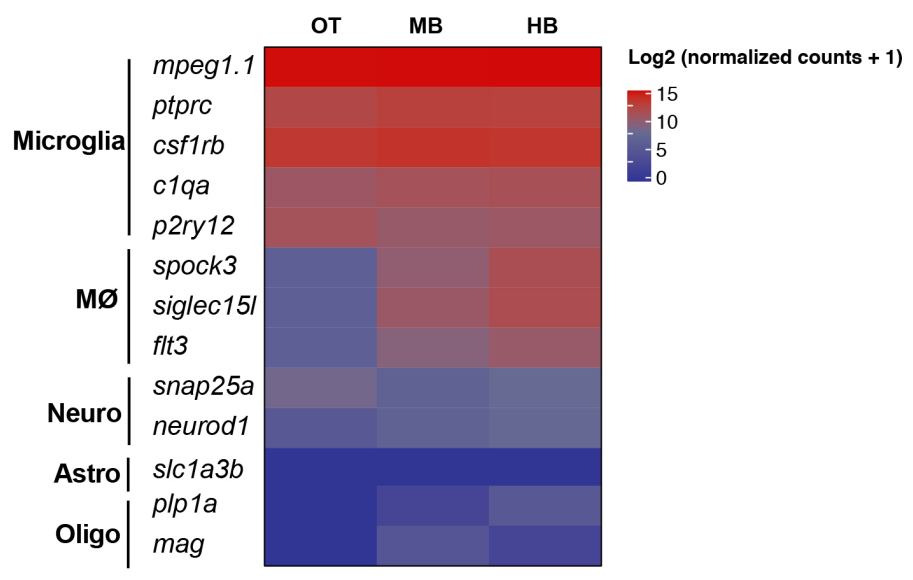

468

Figure S3. Gating strategy and quality control of bulk sequencing data, supplemental to Figure 3.
(A) Gating strategy to isolate mpeg-EGFP+ myeloid cells for region-specific bulk sequencing. (B) Heatmap of selected microglia, macrophage, neuronal, astrocyte, and oligodendrocyte genes. Optic tectum (OT), midbrain (MB), and hindbrain (HB). 
Silva et al.

A
B

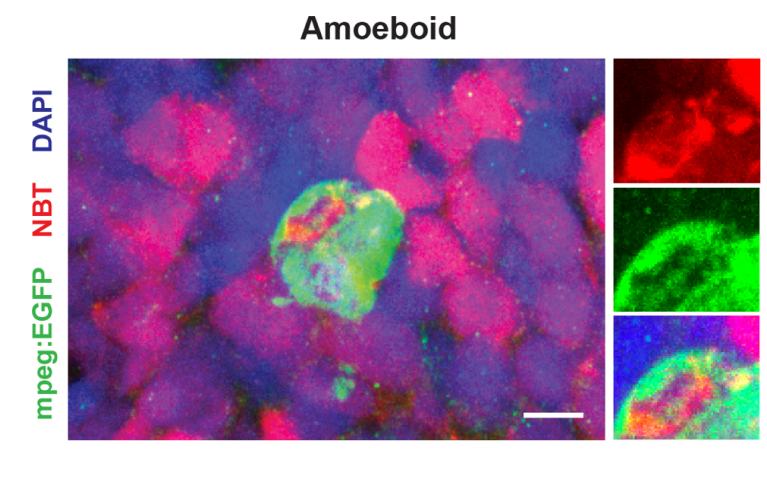

Figure S4. Neuronal engulfment in the OT and HB at $28 \mathrm{dpf}$

(A-B) Representative image and quantification of NBT:DsRed neuronal bodies within ramified and amoeboid microglia (Sphericity > .6) from OT and HB brain regions. Two separate t-test, ${ }^{* * *} p$ $<0.001, n=3$ microglia per fish represented as mean values ( $n=3$ juvenile fish). Scale bar $10 \mu m$.

\section{Supplemental excel files:}

Table S1: Pan hematopoietic single cell clusters in $28 \mathrm{dpf}$ zebrafish brain

Table S2: Myeloid single cell clusters in $28 \mathrm{dpf}$ zebrafish brain

Table S3: Adult and juvenile single cell clusters

Table S4: Region specific myeloid cell profiling in $28 \mathrm{dpf}$ zebrafish brain 
KEY RESOURCES TABLE

519

\begin{tabular}{|c|c|c|}
\hline REAGENT or RESOURCE & SOURCE & IDENTIFIER \\
\hline \multicolumn{3}{|l|}{ Antibodies } \\
\hline Chicken anti-GFP 1:1000 & Aves Labs & $\begin{array}{l}\text { Cat\#GFP-1020, } \\
\text { RRID:AB10000240 }\end{array}$ \\
\hline Rabbit anti-DsRed 1:1000 & Takara Bio & $\begin{array}{l}\text { Cat\#632496;RRID:AB- } \\
10013483\end{array}$ \\
\hline $\begin{array}{l}\text { Mouse anti-synaptic vesicle } \\
\text { glycoprotein 2A (SV2) 1:500 }\end{array}$ & DHSB & RRID: AB_2315387 \\
\hline Rat anti-BrdU 1:500 & Abcam & Cat\#ab6326;RRID:AB_305426 \\
\hline Mouse anti-4c4 1:200 & Gift from Hitchcock Lab & $\begin{array}{l}\text { Cat\#92092321;RRID:ĀB_10013 } \\
752\end{array}$ \\
\hline $\begin{array}{l}\text { Anti-Digoxigenin-AP, Fab } \\
\text { Fragments } 1: 500\end{array}$ & Roche & $\begin{array}{l}\text { Cat\#11093274910,RRID:AB_27 } \\
34716\end{array}$ \\
\hline $\begin{array}{l}\text { Alexa Fluor } 488 \text { goat anti- } \\
\text { chicken 1:500 }\end{array}$ & Thermo Fisher & $\begin{array}{l}\text { Cat\#A- } \\
\text { 11039;RRID:AB_2534096 }\end{array}$ \\
\hline $\begin{array}{l}\text { Alexa Fluor } 555 \text { goat anti-mouse } \\
1: 500\end{array}$ & Thermo Fisher & $\begin{array}{l}\text { Cat\#A-21422, } \\
\text { RRID:AB 141822 }\end{array}$ \\
\hline $\begin{array}{l}\text { Alexa Fluor } 555 \text { goat anti- } \\
\text { rabbit 1:500 }\end{array}$ & Thermo Fisher & $\begin{array}{l}\text { Cat\# A- } \\
\text { 21429;RRID:AB_2535850 }\end{array}$ \\
\hline $\begin{array}{l}\text { Alexa Fluor } 647 \text { goat anti-mouse } \\
1: 500\end{array}$ & Thermo Fisher & $\begin{array}{l}\text { Cat\# A-21235; } \\
\text { RRID:AB_2535804 }\end{array}$ \\
\hline $\begin{array}{l}\text { Alexa Fluor } 647 \text { goat anti- } \\
\text { Rat 1:500 }\end{array}$ & Thermo Fisher & $\begin{array}{l}\text { Cat\# A-21248; } \\
\text { RRID:AB_2535816 }\end{array}$ \\
\hline \multicolumn{3}{|l|}{ Critical Commercial Assays } \\
\hline $\begin{array}{l}\text { Chromium single cell gene } \\
\text { expression platform, version } 3\end{array}$ & 10x Genomics & $\begin{array}{l}\text { Library and gel bead kit-V3, } \\
\text { 120267:Chip B kit:1000009 }\end{array}$ \\
\hline $\begin{array}{l}\text { SIGMAFAST Fast Red } \\
\text { TR/Naphthol AS-MX Tablets }\end{array}$ & Sigma-Aldrich & C\# F4648 \\
\hline $\begin{array}{l}\text { ProSense } 680 \text { Fluorescent } \\
\text { Imagining Agent }\end{array}$ & PerkinElmer & C\#NEV10003 \\
\hline \multicolumn{3}{|l|}{ Deposited Data } \\
\hline $\begin{array}{l}\text { Single cell RNA-Sequencing of } \\
\text { microglia from juvenile and } \\
\text { adults }\end{array}$ & Gene Expression Omnibus & Input information \\
\hline $\begin{array}{l}\text { Bulk RNA-sequencing of } \\
\text { microglia from OT, MB, HB brain } \\
\text { regions }\end{array}$ & Gene Expression Omnibus & Input information \\
\hline \multicolumn{3}{|l|}{$\begin{array}{l}\text { Experimental Models: } \\
\text { Organisms/Strains }\end{array}$} \\
\hline Zebrafish: Tg(mpeg1:EGFP) & 36 & ZFIN: ZDB-ALT-0000 \\
\hline Zebrafish: $\operatorname{Tg}(\mathrm{Cd} 45: D s R e d)$ & 37 & \\
\hline \multicolumn{3}{|l|}{ Oligonucleotides } \\
\hline Cd74a ISH primers, & In house & $\begin{array}{l}\text { F: } \\
\text { TAATACGACTCACTATAGGGG } \\
\text { GACAGGAGAAACTCAAGG;R: } \\
\text { AATTAACCCTCACTAAAGGGC } \\
\text { CATCCCAAACAACATGC }\end{array}$ \\
\hline
\end{tabular}


Silva et al.

\begin{tabular}{|l|l|l|}
\hline & & \\
\hline Ctsba ISH primers, & In house & $\begin{array}{l}\text { F: } \\
\text { TAATACGACTCACTATAGGGA } \\
\text { TGCAAGAGAGCAGTGG; R: } \\
\text { AATTAACCCTCACTAAAGGGA } \\
\text { AGTCCAATGAGCAGGTC }\end{array}$ \\
\hline Software and Algorithms & & \\
\hline Seurat (Version 3.1.4) & 54 & https://satijalab.org/seurat \\
\hline STAR (Version 2.5.4b) & 55 & $\begin{array}{l}\text { https://github.com/alexdobin/ST } \\
\text { AR/releases }\end{array}$ \\
\hline DESeq2 package (Version 4) & 56 & $\begin{array}{l}\text { hhtp://www.bioconductor.org/pa } \\
\text { ckages/release/bioc/html/DESe2 } \\
\text { html }\end{array}$ \\
\hline Harmony (Version 1.0) & & RRID: SCR_003070 \\
\hline ImageJ & & RRID: SCR_007370 \\
\hline Imaris & RRID: SCR_002798 \\
\hline Prism & Oxford Instruments & \\
\hline
\end{tabular}

\section{RESOURCE AVAILABILITY \\ Lead Contact Anna Molofsky (anna.molofsky@ucsf.edu)}

\section{DATA AND CODE AVAILABILITY}

Single cell RNA sequencing and bulk RNA sequencing data were deposited in NCBl's Gene Expression Omnibus (Barrett et al., 2013) under accession number [GSE164772] single cell and [GSE164771] bulk.

\section{EXPERIMENTAL MODEL AND SUBJECT DETAILS}

Wild-type, AB strain zebrafish (Danio rerio; ZIRC, University of Oregon, Eugene, OR) were propagated, maintained, and housed in recirculating habitats at $28.5^{\circ} \mathrm{C}$ and on a $14 / 10-\mathrm{h}$ light/dark cycle. Embryos were collected after natural spawns, incubated at $28.5^{\circ} \mathrm{C}$ and staged by hours post fertilization (hpf). Juveniles used were 28 days of age, a time in development before sex determination. Adults were of either sex were used at 12 months of age. Ages were matched within experiments. The transgenic reporter lines, Tg[CD45:DsRed] and Tg[mpeg1:EGFP] was used to identify hematopoietic lineage and mononuclear phagocytes ${ }^{36,37}$. All animal protocols were approved by and in accordance with the guidelines established by the Institutional Animal Care and Use Committee and Laboratory Animal Resource Center.

\section{METHOD DETAILS}

Immunohistochemistry: Tissues were fixed overnight at $4^{\circ} \mathrm{C}$ in $0.1 \mathrm{M}$ phosphate buffered $4 \%$ paraformaldehyde, cryoprotected with $20 \%$ sucrose, and embedded in optical cutting temperature (OCT) medium (Sakura Finetek USA, Torrance, CA). Immunohistochemistry (IHC) was performed as previously described (Silva et al., 2020). Briefly, 20- $\mu$ m-thick sections were collected and mounted onto glass slides. Sections were washed in phosphate buffer saline with $0.5 \%$ Triton- $\mathrm{X}$ (PBST) and incubated with 20\% heat-inactivated normal goat serum in PBST for 2 hours (NSS; Sigma-Aldrich, Corp.). Primary antibodies were applied overnight at $4^{\circ} \mathrm{C}$. Sections were then washed with PBST and incubated in secondary antibodies for 1 hour at room temperature. Prior 
551

to $\mathrm{IHC}$ for BrdU, sections were immersed in $100^{\circ} \mathrm{C}$ sodium citrate buffer ( $10 \mathrm{mM}$ sodium citrate, $0.05 \%$ Tween $20, \mathrm{pH} 6.0$ ) for 30 minutes and cooled at room temperature for 20 minutes. IHC was performed as described above.

Labeling neurogenic zone: In juveniles, dividing cells were labeled with BrdU by housing animals in system water containing $5 \mathrm{mM}$ BrdU for 24 hours prior to collection ${ }^{57}$.

In situ Hybridization: In situ hybridizations were performed as previously described ${ }^{57}$. Digoxigenin (DIG)-labeled riboprobes for cd74a and ctsba was generated by PCR amplification using primers containing the T3 or T7 promoter sequences.

cd74a (F): 5' TAATACGACTCACTATAGGGGGACAGGAGAAACTCAAGG 3' cd74a-9 (R): 5' AATTAACCCTCACTAAAGGGCCATCCCAAACAACATGC 3'.

ctsba (F): 5' TAATACGACTCACTATAGGGATGCAAGAGAGCAGTGG 3' ctsba (R): 5'

Briefly, 20- $\mu$ m-thick sections were hybridized with riboprobes at $55^{\circ} \mathrm{C}$, incubated with an alkalinephosphatase-conjugated anti-DIG antibody and visualized using Fast Red TR/Naphthol AS-MX (SIGMAFAST) as the enzymatic substrate. When in situ hybridizations were combined with BrdU $\mathrm{IHC}$, sections were removed from the fast red solutions, rinsed and post-fixed in buffered $4 \%$ paraformaldehyde for 10 minutes then processed for BrdU IHC as described above.

ProSense680 injections: Juvenile fish were injected with $2 \mathrm{~nL}$ of ProSense680 at a concentration of $20 \mathrm{nmol}$ directly into the brain and returned to system water immediately following injections. Fish were euthanized 24 hours post injection and processed for immunohistochemistry as stated above.

Microglia morphology quantitation: Z-stack images of mpeg:EGFP and 4C4 staining were acquired at the step size of $.5 \mu \mathrm{m}$ using a $63 x$ objective (NA 1.4) on an LSM 800 Confocal Microscope (Zeiss) for a thickness of $20 \mu \mathrm{m}$. Microglia sphericity was quantified using Imaris software (Bitplane) by creating a 3D surface reconstruction of mpeg:EGFP ${ }^{+}$microglia that were also positive for 4c4. All images were set to a standard threshold to accurately maintain morphology for quantifications.

Microglia engulfment assay: Images were acquired with an LSM 800 Confocal Microscope (Zeiss) using the same parameters as described above. Imaris software (Bitplane) was used to generate 3D surface rendering of microglia, which were then masked for NBT:DsRed or SV2 channels within that microglia. Masked channels were then 3D rendered to obtain volume data. NBT:DsRed and SV2 engulfment was calculated per cell as the volume of SV2 divided by the volume of the microglia.

Fluorescence activated cell sorting (FACS): For bulk RNA-sequencing of juvenile $28 \mathrm{dpf}$ Tg(mpeg1:EGFP) zebrafish, the optic tectum, midbrain and hindbrain were dissected (10 zebrafish were pooled per sample). For scRNA-sequencing of $28 \mathrm{dpf} T g$ (mpeg1:EGFP): $\operatorname{Tg}(C d 45: D s R e d)$ juveniles (10 zebrafish pooled per lane) and 1 year old $T g$ (mpeg1:EGFP): $\mathrm{Tg}(\mathrm{Cd} 45: D s R e d)$ adults (3 zebrafish pooled per lane) whole brains were dissected. To isolate microglia and other CD45+ cells, we followed a previously described method. Briefly, the brain(s) (regions) were mechanically dissociated in isolation medium (1x HBSS, 0.6\% glucose, $15 \mathrm{mM}$ HEPES, 1 mM EDTA pH 8.0) using a glass tissue homogenizer (VWR). Subsequently, the cell suspension was filtered through a $70 \mu \mathrm{m}$ filter (Falcon) and pelleted at $300 \mathrm{~g}, 4^{\circ} \mathrm{C}$ for 10 minutes. 
602

603

604

605

606

607

608

609

610

611

612

613

614

615

616

617

618

619

620

621

622

623

624

625

626

627

628

629

630

631

632

633

634

635

636

637

638

639

640

641

642

643

The pellet was resuspended in $22 \%$ Percoll (GE Healthcare) and centrifuged at $900 \mathrm{~g}, 4^{\circ} \mathrm{C}$ for 20 minutes (acceleration set to 4 and deceleration set to 1). Afterwards, the myelin free pellet was resuspended in isolation medium that did not contain phenol red. Prior to sorting on a BD FACS Aria III, cell suspension was incubated with DAPI (Sigma). For bulk RNA-sequencing, microglia were gated on FSC/SSC scatter, live cells by DAPI, and Mpeg1-GFP+. After sorting, cells were spun down at $500 \mathrm{~g}, 4^{\circ} \mathrm{C}$ for $10 \mathrm{~min}$ and the pellet was lysed with RLT+ (Qiagen). For scRNAsequencing, microglia, macrophages and CD45+ cells were collected by gating on FSC/SSC scatter, live cells by DAPI, and all CD45-DsRed (which included both mpeg+ and negative subsets). After sorting, cells were spun down at $500 \mathrm{~g}, 4^{\circ} \mathrm{C}$ for $10 \mathrm{~min}$ and resuspended in PBS $+0.05 \%$ BSA (Sigma).

Bulk RNA-sequencing of microglia: RNA was extracted using the RNeasy® Plus Micro kit (Qiagen) from RLT+ lysed microglia. RNA quality and concentration were measured using the Agilent RNA 6000 Pico kit on an Agilent Bioanalyzer. All samples had an RNA Integrity Number $(\mathrm{RIN})>8$. For each sample, a total of $10 \mathrm{ng}$ of RNA was loaded as input for cDNA amplification and library construction using the QuantSeq 3' mRNA-Seq Library Prep Kit FWD for Illumina (Lexogen) following manufacturer's instructions. Library quality was determined with the Agilent High Sensitivity DNA kit on an Agilent Bioanalyzer and concentrations measured with the Qubit ${ }^{\mathrm{TM}}$ dsDNA HS Assay Kit (Thermo Fisher) on a Qubit ${ }^{\mathrm{TM}}$ (Thermo Fisher). Library pools were singleend (65-bp reads) sequenced on two lanes using an Illumina HiSeq 4000 yielding 40-50 million reads per sample.

Bulk RNA-sequencing analysis: Quality of reads was assessed using FastQC (http://www.bioinformatics.babraham.ac.uk/projects/fastqc). All samples passed quality control and reads were aligned to Danio rerio GRCz11.98 genome (retrieved from Ensemble) using STAR (version 2.5.4b) ${ }^{55}$ with '--outFilterMultimapNmax 1' to only keep uniquely mapped reads. Uniquely mapped reads were counted using $\mathrm{HTSeq}^{58}$. Subsequently, the DESeq2 package ${ }^{56}$ in $R$ software was used to normalize the raw counts and perform differential gene expression analysis. Batch correction was done using the Limma package ${ }^{59}$ and heatmaps were made using ComplexHeatmap ${ }^{60}$ package in $\mathrm{R}$ software. Metascape was used for gene ontology analysis ${ }^{61}$.

Single cell RNA-sequencing: Single cells were isolated as described above. Approximately 15,000 cells were loaded into each well of Chromium Chip B (v3), libraries were prepared using the 10x Genomic Chromium 3' Gene Expression Kit in-house as described in the Manual and sequenced on two lanes of the NovaSeq SP100 sequencer for an average depth of 30,00050,000 reads per cell. BCL files are converted to Fastq, then used as inputs to the 10X Genomics Cell Ranger pipeline. Samples were processed using the Cell Ranger 2.1 pipeline and aligned to the GRCz11.94 (danRer11) zebrafish reference genome. Clustering and differential expression analysis were conducted using Seurat version 3.1.4. for figure 3 (juvenile) and figure 5 (adult) data.

Feature thresholds

\begin{tabular}{|l|l|l|}
\hline & Whole Brain Juvenile Isolation & Whole Brain Adult Isolation \\
\hline $\begin{array}{l}\text { Number of } \\
\text { cells }\end{array}$ & $\begin{array}{l}\text { Loaded: 20,000 } \\
\text { Total passing QC: } 6666 \\
\text { Mpeg+: } 3539\end{array}$ & $\begin{array}{l}\text { Loaded: 10,000 } \\
\text { Mpeg+ after QC: } 2336\end{array}$ \\
\hline $\begin{array}{l}\text { Biological } \\
\text { replicates }\end{array}$ & 10 fish & 3 fish \\
\hline $10 x$ lanes & 2 & 1 \\
\hline
\end{tabular}


Silva et al.

644

645

646

647

648

649

650

651

652

653

654

655

656

657

658

659

660

661

662

663

664

665

666

667

668

669

670

671

672

673

674

675

676

677

678

679

680

681

682

683

684

685

686

687

688

689
Following alignment in Cell Ranger as described above, counts were imported into $\mathrm{R}$ and analyzed using the Seurat package (Butler et al., 2018; Satija et al., 2015). Cells outside of the thresholds listed in the table were excluded from downstream analysis.

Counts were then normalized, regressing out percent mitochondrial RNA and total counts. The top 6000 most variable genes were used to calculate 50 principal components, and the top 30 PCs were used for nearest neighbor, UMAP, and cluster calculations with the resolutions shown in the table. Individual cell types were identified through calculation of marker genes using the MAST test for genes expressed in at least $20 \%$ of cells in the cluster and a log fold change of 0.2 or greater and adjusted $p$ value less than $10^{\wedge}-8$. The microglial and macrophage clusters were isolated based on expression of CD45 (ptprc) and mpeg1.1. Normalization, clustering, and differential gene expression was recalculated for each sample (juvenile, juvenile mpeg+, adult and juvenile mpeg+). GO analysis was conducted using the Metascape webpage (www.metascape.org). Adult and juvenile datasets were combined using Harmony 1.0. Bulk vs single cell analysis was conducted using the following thresholds for bulk sequencing results: adjp $<0.05$, Ifc $>1.2$, basemean $>100$. Following thresholding, only genes uniquely enriched in one region were used to calculate eigengene values.

\section{QUANTIFICATION AND STATISTICAL ANALYSIS}

Graphpad Prism 8.3.0 was used for all histological quantification analyses. Statistical tests are described in text and figure legends. RNA-sequencing data was analyzed in R as described above. 
Silva et al.

701

702

703

704

705

706

707

708

709

710

711

712

713

714

715

716

717

718

719

720

721

722

723

724

725

726

727

728

729

730

731

\section{REFERENCES}

1. Colonna, M. \& Butovsky, O. Microglia function in the central nervous system during health and neurodegeneration. Annu. Rev. Immunol. 35, 441-468 (2017).

2. Wu, Y., Dissing-Olesen, L., MacVicar, B. A. \& Stevens, B. Microglia: Dynamic Mediators of Synapse Development and Plasticity. Trends in Immunology vol. 36 605-613 (2015).

3. Li, Q. et al. Developmental Heterogeneity of Microglia and Brain Myeloid Cells Revealed by Deep Single-Cell RNA Sequencing. Neuron 101, 207-223.e10 (2019).

4. Hammond, T. R. et al. Single-Cell RNA Sequencing of Microglia throughout the Mouse Lifespan and in the Injured Brain Reveals Complex Cell-State Changes. Immunity 50, 253-271.e6 (2019).

5. Ayata, P. et al. Epigenetic regulation of brain region-specific microglia clearance activity. Nat. Neurosci. 21, 1049-1060 (2018).

6. Sierra, A. et al. Microglia shape adult hippocampal neurogenesis through apoptosiscoupled phagocytosis. Cell Stem Cell 7, 483-495 (2010).

7. VanRyzin, J. W. et al. Microglial Phagocytosis of Newborn Cells Is Induced by Endocannabinoids and Sculpts Sex Differences in Juvenile Rat Social Play. Neuron 102, 435-449.e6 (2019).

8. Sakai, C., ljaz, S. \& Hoffman, E. J. Zebrafish Models of Neurodevelopmental Disorders: Past, Present, and Future. Front. Mol. Neurosci. 11, (2018).

9. Oosterhof, N., Boddeke, E. \& van Ham, T. J. Immune cell dynamics in the CNS: Learning from the zebrafish. GLIA vol. 63 719-735 (2015).

10. Martins, R. R., Ellis, P. S., MacDonald, R. B., Richardson, R. J. \& Henriques, C. M. Resident Immunity in Tissue Repair and Maintenance: The Zebrafish Model Coming of Age. Front. Cell Dev. Biol. 7, (2019).

11. Lyons, D. A. \& Talbot, W. S. Glial cell development and function in zebrafish. Cold Spring Harb. Perspect. Biol. 7, 1-22 (2015).

12. Lyons, D. A. \& Talbot, W. S. Glial cell development and function in zebrafish. Cold Spring Harb. Perspect. Biol. 7, a020586 (2014).

13. Chen, J., Poskanzer, K. E., Freeman, M. R. \& Monk, K. R. Live-imaging of astrocyte morphogenesis and function in zebrafish neural circuits. Nat. Neurosci. 23, 1297-1306 (2020).

14. Kawai, H., Arata, N. \& Nakayasu, H. Three-dimensional distribution of astrocytes in zebrafish spinal cord. Glia 36, 406-413 (2001).

15. Meeker, N. D. \& Trede, N. S. Immunology and zebrafish: Spawning new models of human disease. Dev. Comp. Immunol. 32, 745-757 (2008).

16. Renshaw, S. A. \& Trede, N. S. A model 450 million years in the making: Zebrafish and vertebrate immunity. DMM Dis. Model. Mech. 5, 38-47 (2012).

17. Rougeot, J. et al. RNAseq Profiling of Leukocyte Populations in Zebrafish Larvae Reveals a cxcl11 Chemokine Gene as a Marker of Macrophage Polarization During Mycobacterial Infection. Front. Immunol. 10, 832 (2019).

18. Chen, J. et al. Cerebrovascular Injuries Induce Lymphatic Invasion into Brain 
Parenchyma to Guide Vascular Regeneration in Zebrafish. Dev. Cell 49, 697-710.e5 (2019).

19. Fleming, A., Diekmann, H. \& Goldsmith, P. Functional Characterisation of the Maturation of the Blood-Brain Barrier in Larval Zebrafish. PLoS One 8, e77548 (2013).

20. Villani, A. et al. Clearance by Microglia Depends on Packaging of Phagosomes into a Unique Cellular Compartment. Dev. Cell 49, 77-88.e7 (2019).

21. Mazaheri, F. et al. Distinct roles for BAI1 and TIM-4 in the engulfment of dying neurons by microglia. Nat. Commun. 5, (2014).

22. Shen, K., Sidik, H. \& Talbot, W. S. The Rag-Ragulator Complex Regulates Lysosome Function and Phagocytic Flux in Microglia. Cell Rep. 14, 547-559 (2016).

23. Wu, S. et al. II34-Csf1r Pathway Regulates the Migration and Colonization of Microglial Precursors. Dev. Cell 46, 552-563.e4 (2018).

24. Svahn, A. J. et al. Development of ramified microglia from early macrophages in the zebrafish optic tectum. Dev. Neurobiol. 73, 60-71 (2013).

25. Casano, A. M., Albert, M. \& Peri, F. Developmental Apoptosis Mediates Entry and Positioning of Microglia in the Zebrafish Brain. Cell Rep. 16, 897-906 (2016).

26. Herbomel, P., Thisse, B. \& Thisse, C. Zebrafish early macrophages colonize cephalic mesenchyme and developing brain, retina, and epidermis through a M-CSF receptordependent invasive process. Dev. Biol. 238, 274-288 (2001).

27. Hughes, A. N. \& Appel, B. Microglia phagocytose myelin sheaths to modify developmental myelination. Nat. Neurosci. 23, 1055-1066 (2020).

28. Oosterhof, N. et al. Colony-Stimulating Factor 1 Receptor (CSF1R) Regulates Microglia Density and Distribution, but Not Microglia Differentiation In Vivo. Cell Rep. 24, 12031217.e6 (2018).

29. Xu, J. et al. Temporal-Spatial Resolution Fate Mapping Reveals Distinct Origins for Embryonic and Adult Microglia in Zebrafish. Dev. Cell 34, 632-641 (2015).

30. Ferrero, G. et al. Embryonic Microglia Derive from Primitive Macrophages and Are Replaced by cmyb-Dependent Definitive Microglia in Zebrafish. Cell Rep. 24, 130-141 (2018).

31. Wu, S. et al. Two phenotypically and functionally distinct microglial populations in adult zebrafish. Sci. Adv vol. 6 http://advances.sciencemag.org/ (2020).

32. Butovsky, O. et al. Identification of a unique TGF- $\beta$-dependent molecular and functional signature in microglia. Nat. Neurosci. 17, 131-143 (2014).

33. Geirsdottir, L. et al. Cross-Species Single-Cell Analysis Reveals Divergence of the Primate Microglia Program. Cell 179, 1609-1622.e16 (2019).

34. Mazzolini, J. et al. Gene expression profiling reveals a conserved microglia signature in larval zebrafish. Glia 68, 298-315 (2020).

35. Oosterhof, N. et al. Identification of a conserved and acute neurodegeneration-specific microglial transcriptome in the zebrafish. Glia 65, 138-149 (2017).

36. Ellett, F., Pase, L., Hayman, J. W., Andrianopoulos, A. \& Lieschke, G. J. mpeg1 promoter transgenes direct macrophage-lineage expression in zebrafish. Blood 117, e49-e56 (2011). 
37. Wittamer, V., Bertrand, J. Y., Gutschow, P. W. \& Traver, D. Characterization of the mononuclear phagocyte system in zebrafish. Blood 117, 7126-7135 (2011).

38. Hernández, P. P. et al. I N N A T E L Y M P H O I C E L L S Single-cell transcriptional analysis reveals ILC-like cells in zebrafish. Sci. Immunol vol. 3 http://immunology.sciencemag.org/ (2018).

39. Tang, Q. et al. Dissecting hematopoietic and renal cell heterogeneity in adult zebrafish at single-cell resolution using RNA sequencing. J. Exp. Med. 214, 2875-2887 (2017).

40. Kasheta, M. et al. Identification and characterization of T reg-like cells in zebrafish. J. Exp. Med. 214, 3519-3530 (2017).

41. Liu, X. et al. Zebrafish B Cell Development without a Pre-B Cell Stage, Revealed by CD79 Fluorescence Reporter Transgenes. J. Immunol. 199, 1706-1715 (2017).

42. Korsunsky, I. et al. Fast, sensitive and accurate integration of single-cell data with Harmony. Nat. Methods 16, 1289-1296 (2019).

43. Fonseca, M. I. et al. Cell-specific deletion of C1qa identifies microglia as the dominant source of C1q in mouse brain. J. Neuroinflammation 14, 1-15 (2017).

44. Stephan, A. H., Barres, B. A. \& Stevens, B. The complement system: An unexpected role in synaptic pruning during development and disease. Annu. Rev. Neurosci. 35, 369-389 (2012).

45. Ndoja, A. et al. Ubiquitin Ligase COP1 Suppresses Neuroinflammation by Degrading c/EBP $\beta$ in Microglia. Cell 182, 1156-1169.e12 (2020).

46. Lui, H. et al. Progranulin Deficiency Promotes Circuit-Specific Synaptic Pruning by Microglia via Complement Activation. Cell 165, 921-935 (2016).

47. Weissleder, R., Tung, C. H., Mahmood, U. \& Bogdanov, A. In vivo imaging of tumors with protease-activated near-infrared fluorescent probes. Nat. Biotechnol. 17, 375-378 (1999).

48. Wolf, S. A., Boddeke, H. W. G. M. \& Kettenmann, H. Microglia in Physiology and Disease. Annu. Rev. Physiol. 79, 619-643 (2017).

49. Nguyen, P. T. et al. Microglial Remodeling of the Extracellular Matrix Promotes Synapse Plasticity. Cell 182, 388-403.e15 (2020).

50. Badimon, A. et al. Negative feedback control of neuronal activity by microglia. Nature 586, 417-423 (2020).

51. Li, Y., Du, X. F., Liu, C. S., Wen, Z. L. \& Du, J. L. Reciprocal Regulation between Resting Microglial Dynamics and Neuronal Activity In Vivo. Dev. Cell 23, 1189-1202 (2012).

52. Stevens, B. et al. The Classical Complement Cascade Mediates CNS Synapse Elimination. Cell 131, 1164-1178 (2007).

53. Mathys, H. et al. Temporal Tracking of Microglia Activation in Neurodegeneration at Single-Cell Resolution. Cell Rep. 21, 366-380 (2017).

54. Satija, R., Farrell, J. A., Gennert, D., Schier, A. F. \& Regev, A. Spatial reconstruction of single-cell gene expression data. Nat. Biotechnol. 33, 495-502 (2015).

55. Dobin, A. et al. STAR: Ultrafast universal RNA-seq aligner. Bioinformatics 29, 15-21 (2013).

56. Love, M. I., Huber, W. \& Anders, S. Moderated estimation of fold change and dispersion for RNA-seq data with DESeq2. Genome Biol. 15, (2014). 


\section{Silva et al.}

816

817

818

819

820

821

822

823

824

825

826
57. Silva, N. J. et al. Inflammation and matrix metalloproteinase 9 (Mmp-9) regulate photoreceptor regeneration in adult zebrafish. Glia 68, 1445-1465 (2020).

58. Anders, S., Pyl, P. T. \& Huber, W. HTSeq-A Python framework to work with highthroughput sequencing data. Bioinformatics 31, 166-169 (2015).

59. Ritchie, M. E. et al. Limma powers differential expression analyses for RNA-sequencing and microarray studies. Nucleic Acids Res. 43, e47 (2015).

60. Gu, Z., Eils, R. \& Schlesner, M. Complex heatmaps reveal patterns and correlations in multidimensional genomic data. Bioinformatics 32, 2847-2849 (2016).

61. Zhou, Y. et al. Metascape provides a biologist-oriented resource for the analysis of systems-level datasets. Nat. Commun. 10, (2019). 